DePt. of Math. Univ. of Oslo

Pure Mathematics No. 24

ISSN 0806-2439 September 2005

\title{
STOCHASTIC MODELING OF FINANCIAL ELECTRICITY CONTRACTS
}

\author{
FRED ESPEN BENTH AND STEEN KOEKEBAKKER
}

\begin{abstract}
We discuss the modeling of electricity contracts traded in many deregulated power markets. These forward/futures type contracts deliver (either physically or financially) electricity over a specified time period, and is frequently referred to as swaps since they in effect represent an exchange of fixed for floating electricity price. We provide a thorough discussion of how the Nordic power market Nord Pool is organized, in order to motivate a Heath-Jarrow-Morton approach to model swap prices since the notion of a spot price is not easily defined. For general stochastic dynamical models, we connect the spot price, the fixed-delivery forward price and the swap price, and analyze two different ways to apply the Heath-Jarrow-Morton approach to swap pricing: Either one specifies a dynamics for the non-existing fixed-delivery forwards and derives the implied swap dynamics, or one models directly on the swaps. The former is shown to lead to quite complicated stochastic models for the swap price, even when the forward dynamics is simple. The latter has some theoretical problems due to a no-arbitrage condition that has to be satisfied for swaps with overlapping delivery period. To overcome this problem, we suggest a practical modeling approach where we suppose that the market only consists of non-overlapping swaps, and model these directly. A thorough empirical study is performed using data collected from Nord Pool. Our investigations demonstrate that it is possible to state reasonable models for the swap price dynamics which is analytically tractable for risk management and option pricing purposes, however, this is an area of further research.
\end{abstract}

\section{INTRODUCTION}

This paper considers the problem of modeling the pricing dynamics of forward and futures contracts traded in electricity markets. Forward and futures contracts in the electricity market have the distinctive feature of guaranteeing delivery of the underlying commodity over a period rather than at a fixed future time. We propose and study the Heath-Jarrow-Morton (HJM) approach to model the pricing dynamics of such average-type

Date: September 15, 2005.

Key words and phrases. Electricity markets, Nord Pool, forwards and futures, swaps, Heath-JarrowMorton approach, Lévy processes.

We are grateful to Fridthjof Ollmar for providing us with data from the Nord Pool power exchange. 
forward and futures contracts. Empirical tests using market information from the Nordic power exchange Nord Pool are provided for certain lognormal swap models.

In stochastic modeling of fixed-income markets, one can roughly divide the different approaches into two (see e.g. Musiela and Rutkowski [44]): one either starts out with a stochastic model for the spot interest rate, and from this one derives bond prices based on no-arbitrage principles. The alternative to this is to apply the HJM approach suggested by Heath, Jarrow and Morton [26] to specify the complete yield curve dynamics (that is, the forward rates) directly. The former approach takes the point of view that all activity in the fixed-income markets can be explained by one (or a finite number of) factor(s), while, at the other extreme, the HJM approach uses infinitely many factors. For commodities there is a trade in a spot and forward market, and the modeling paradigms from fixedincome theory are adopted (see e.g. Clewlow and Strickland [13]). Unlike the spot rate in fixed-income markets, it is possible to trade commodities on a spot market. However, the best liquidity is usually found in the market for forward and futures contracts due to the storage and transaction costs of most commodities. From general arbitrage theory the forward price can be derived as the risk-neutral expected present value of the spot price at the delivery time, assuming a liquid market for the spot (like, e.g., stock markets). For commodities, the frictions in the spot market has introduced the concept of convenience yield, measuring the benefit from ownership of the commodity (see e.g., Hull [30]).

Turning our attention to electricity markets, most of the existing literature focuses on developing realistic spot price models (see e.g., Clewlow and Strickland [13] and Pilipovic [46]). Based on a stochastic model for the time evolution of the electricity spot price, one can derive the forward and futures price dynamics by appealing to the arbitrage theory. For instance, Lucia and Schwartz [42] suggest a mean-reverting stochastic process with multiplicative noise, together with different multi-factor extensions motivated from Schwartz [50], to model the electricity spot dynamics. Their models are fitted to data collected from Nord Pool, and prices for the forward and futures contracts are derived. Geman and Roncoroni [23] build up a similar model allowing for spikes via jump process, and demonstrate the flexibility of their approach by a successful fitting to observed spot prices collected from several markets. Other works on spot price models include, to mention a few, Barlow [2], Benth et al. [4], Burger et al. [11], Cartea and Figueroa [12], Elliott, Sick and Stein [17], Kaaresen and Husby [32] and Vehviläinen and Pyykkönnen [56].

The spot electricity is not a tradeable asset in the classical sense, since it is non-storable. Moreover, as we will discuss in Section 2, it is not easy to give a precise definition to what exactly is the spot in the electricity market. The basic tradeable assets in the electricity markets can typically be described as forward and futures contracts with delivery of electricity over a time period. The main objective of this paper is therefore to introduce and analyse mathematically and empirically stochastic models specified directly on the forward 
and futures contracts traded in the electricity market. We use the HJM-modeling paradigm to define a stochastic dynamics on the price evolution of forward and futures contracts with delivery of electricity over a period. The market for such products with a financial settlement is very liquid, and futhermore, the derivatives market has these contracts as the underlying assets. Moreover, for risk management purposes, these average-type forward and futures contracts are the most interesting ones for participants in the electricity market. From the nature of the contracts, one often refers to them as swaps. From now on we will stick to this name when referring to the forwards and futures traded in the electricity markets, that is, we will call forwards and futures with delivery over a specified period for swaps.

Bjerksund, Rasmussen and Stensland [9] were the first to consider the HJM-approach in electricity markets. They started out with a lognormal model for forward contracts with delivery at fixed times. Such forwards are not traded in the market, so when fitting the model to data one needs to create a forward curve from the quoted swap prices. Furthermore, since the swap can be represented as the integral over the delivery period of such forwards, the lognormality is lost since in general one can not expect an integral of lognormal variables to be lognormal. To have an analytically tractable model for option pricing and risk management purposes, Bjerksund et al. [9] suggest an approximation which associates the volatility in a lognormal specification of the swap to the fitted volatility of the forward. Their procedure of pinning down a swap model involves two approximative steps, where the first involves a price reconstruction of non-traded forward quotes from the traded swap prices, and the second involves an approximation of the derived swap dynamics with a lognormal process. In the present paper we use the stochastic Fubini theorem in order to derive an explicit dynamics for the swap contracts from a stochastic specification of the artificial forwards with fixed delivery times. Our main finding in this respect is that the swap dynamics does not even become Markovian even for natural lognormal specifications of the forwards, unless interpreted as an infinite-dimensional stochastic process.

A more natural point of view when using the HJM-framework is to specify a dynamics directly on the swap price dynamics. Taking the perspective of HJM literally, one should specify an arbitrage-free pricing dynamics for all possible delivery periods. However, since we have contracts with overlapping time intervals for delivery, this will lead to rather restrictive conditions on the dynamics. For instance, in the market, one may have 12 swaps with delivery in each month of next year, and one swap with delivery over the whole of the coming year. This leads us to a no-arbitrage condition saying that any (weighted) combination of swaps with disjoint deliveries must coincide in price with the swap having delivery over the union of these delivery periods. In the limit, we show that in fact forward prices must exist being swaps with delivery over an infinitesimal time period, and the integral over these forwards must coincide with the swap price. This relation is rather strong, and choosing a lognormal dynamics for the swap will give us a possible arbitrage. 
In fact, it seems rather difficult to define a natural swap dynamics which satisfies the no-arbitrage condition in this modeling framework.

As a compromise resolving the problem of specifying directly a swap dynamics, we propose to model only those swaps which do not overlap. Indeed, there is a trade in overlapping swap contracts in the electricity market, however, these constitute only a minor part of the total number of traded swaps. Hence, it seems like a natural approach to assume that we have a specified dynamics, possibly lognormal, only for the swaps which are not decomposable by other swaps with shorter delivery periods. The advantages in having a model for the swap prices directly are several. First of all, we avoid the data smoothing problem encountered by Bjerksund et al. [9], and can utilize the quoted prices directly in model fitting. Furthermore, since most of the traded options on electricity are written on swap contracts as the underlying security, analytical prices are within reach, in particular for plain vanilla options when the swap price dynamics is assumed lognormal, which yields a Black-76 type option pricing formula. Finally, risk management measures like the greeks of options or VaR measures on portfolios are simple with an explicit stochastic dynamics for the swaps. Using spot models, or the framework of Bjerksund et al. [9], may give rather complicated expressions for risk management and option pricing, where numerical methods are called for.

In the practical applications of our analysis we shall focus on lognormal models. The motivation for considering this class of stochastic processes comes from the wish to have a class of models which is analytically tractable from a risk management and option pricing point of view, as well as being reasonably realistic model for the observed data. We suggest different volatility structures for the swap price dynamics. These volatility functions are derived from corresponding fixed-time delivery forwards suggested for other commodity markets. An empirical study of the different models is presented, where we have used a large collection of historical swap price data quoted on the Nord Pool. Based on maximum likelihood techniques, we estimate different volatility structures, and compare their statistical fit via the the log-likelihood value.

In this paper we restrict our attention to continuous diffusions when doing empirical studies of our proposed swap dynamics. The models will involve only one Brownian motion (although our theoretical considerations allow for many), which means that we do empirical studies under a one-factor hypothesis. Of course, this is not very reasonable, and more complex models are called for (even though our fit is rather good). The objective of this paper is not to perform an exhaustive empirical study of swap modelling, but to enlighten our approach. A possible extension could be a model which allows for jumps, and we describe such a model theoretically. However, as Keppo et al. [35] indicates, one should introduce several Brownian motions rather than including a jump process to fully explain the dynamical behaviour of the swap price. We will follow this path in future research. 
The main drawback of our approach is that we can not associate a spot dynamics from the defined swap dynamics. Doing so would imply that the model allowed for a forward price dynamics, which is not possible when considering a lognormal specification of the swap. In practice, however, it is not unlikely that there is a gap between the spot market and the swap market which makes it natural to model the two markets separably, however, with some sort of correlation structure. Another interpretation of the lack of a spot dynamics is that the spot market of electricity is in fact a swap market. Buying on the spot market means that you will receive electricity delivered over a specified forthcoming time period. This is the nature of all electricity trading. Spot trading simply implies that both time to delivery and the length of the delivery period is very short. Our modeling approach has potential in other markets as well, typically in markets were there is a liquid swap market.

Our paper is organized as follows: in Section 2 we describe the market mechanisms in a deregulated electricity market and the physical and financial electricity contracts that are traded. We focus in particular on financial contracts traded in the Nordic market. Previous literature is presented. We also give a brief review of some of the issues dealt with in the literature focusing on this market. Section 3 states the assumptions of our theoretical framework. In Section 4 we start out with a fixed-delivery forward curve dynamics, and show that the implied swap dynamics is complicated, even for simple multiplicative models. In section 5 we specify an arbitrage free model for the swap dynamics prior to the delivery period. We consider a model which is valid for swaps with all possible delivery periods. It turns out that this level of generality implies a quite restrictive class of arbitrage-free models. Section 6 suggest a swap market model for the electricity market. Here we restrict our efforts to modeling non-overlapping swaps. we refer to these basic building blocks as atomic swaps. This model specification allows us to state simple arbitrage free dynamical models for the spot price. In Section 7 we derive call and put option prices for our different swap models, and in Section 8 we estimate different lognormal specifications of the swap market model suggested in Section 6 using data from swap contracts traded on Nord Pool in the period 1995-2004. Our swap market model allows us to use contract prices directly in our estimation procedure and the empirical results suggest that volatility is a function of both season, the length of the delivery period and time to delivery. Several avenues of future theoretical and empirical research is suggested. Finally, some concluding remarks are given in Section 9 .

\section{Description of the Nord Pool market for Electricity}

Electricity is usually labeled as a commodity, although its non-storeability has a profound effect on the infrastructure and the organisation of the electricity market compared with 
other commodity markets. ${ }^{1}$ Electrical power is only useful for practical purposes if it can be delivered during a period of time. This is why electricity has been called a flow commmodity. Deregulated power markets have market mechanisms to balance supply and demand, where electricity is traded in an auction system for standardised contracts. All contracts guarantee the delivery of a given amount of electricity energy for a specified future time period. Some contracts prescribe physical delivery, while others are financially settled.

Financial power contracts are linked to some reference electricity spot price, and they are settled in cash. The market for such contracts is open to speculators, since it is not required to have consumption or production of electricity to participate in the market. We will focus our modeling efforts on the Nordic power market Nord Pool, but our results can be applied to contracts traded at other power exchanges as well. ${ }^{2}$ In the following subsections we will describe both the physical and the financial electricity contracts traded at Nord Pool, along with a brief review of some of the relevant literature connected to the modeling of electricity prices.

2.1. Electricity contracts with physical delivery. By physical electricity contracts we mean contracts with actual consumption or production as part of contract fulfillment. Since capacity is restricted, and the supply and demand must balance, these markets must be supervised by a transmission system operator (TSO). Moreover, typically, the players in these markets are restricted to those with proper facilities for production or consumption. The contracts for physical delivery is usually organised in two different markets, the real time and day ahead market. ${ }^{3}$ This is known as the two-settlement system.

2.1.1. The real time market. The real time (henceforth RT) market is organised by a system operator for short term upward or downward regulation. The auction specifies both load and time period for generation or consumption. Bids in the RT market are submitted to the TSO. Bids may be posted or changed close to the operational time, in accordance with agreed rules. Real-time market bids are for upward regulation (increased generation or reduced consumption) and downward regulation (decreased generation or increased consumption). Both demand-side and supply-side bids are posted, stating prices and volumes. Market participants must be able to commit significant power volumes on short notice. In the Nordic market the TSOs are Statnett (Norway), Svenska Kraftnät

\footnotetext{
${ }^{1}$ Stoft [54] provides a unique treatment of the interplay between economics and engineering in deregulated electricity markets. Wolak [58] gives a description of world wide electricity market organisation after deregulation.

${ }^{2}$ For instance the base load financial contracts traded on the German exchange EEX and the French exchange POWERNEXT are more or less identical to the Nord Pool contracts.

${ }^{3}$ See part 3 in the book by Stoft [54] for a detailed description of alternative market architectures for both day-ahead and real time markets.
} 
(Sweden), Fingrid (Suomen Kataverkko Oyj) (Finland), Elkraft System AS (Zealand Eastern Denmark) and Eltra (Jutland/Funen - Western Denmark). TSOs list bids for each hour in priority order, according to price (merit order), and the merit order for each hour is used to balance the power system. Upward regulation is applied to resolve a grid power deficit; the real-time market price is set at the highest price of the units called upon from the merit order. In the case of grid power surplus, downward regulation is applied; the lowest price of the units called upon from the participation list sets the real-time price. The auctions in each country are in effect Walrasian auctions, but the specific rules for determining the hourly price of power imbalances, based on the real-time market price, differs among the Nordic TSOs. In addition to the RT auction market the national TSOs have established markets for various necessary ancillary services providing balance power to market actors and securing the operational reserves needed for the system balance.

2.1.2. The day-ahead market. There also exists a day-ahead (henceforth DA) market in most deregulated electricity markets. In the Nordic market, the DA market is a nonmandatory market called Elspot and it is organised by Nord Pool. Powernext in France and EEX in Germany are also non-mandatory DA markets, contrary to for instance Omel in Spain. On Elspot, hourly power contracts are traded daily for physical delivery in the next day's 24-hour period (midnight to midnight). On Nord Pool's spot market, Norwegian, Swedish, Finnish and Danish players trade in hourly contracts for each of the 24 hours of the coming day. Each morning, the players submit their bids for purchasing or selling a certain volume of electricity for the different hours of the following day. Once the spot market is closed for bids, at noon each day, the day-ahead price is derived for each hour next day. The day-ahead price is called the system price, and is common to all Nordic countries. In case of congestion due to capacity constraints, the Nordic market is divided into different bidding areas, resulting in zonal prices. Each contract is assigned a specific load for a given future delivery period. This means that, strictly speaking, the DA market is trading in electricity forward contracts with delivery over a specified hour the next day. Figure 1 shows time series of weekly averages of the system price in the Nordic market.

There also exists a market that somewhat closes the gap between the DA and RT markets. This is called the Elbas market. The time span between the Elspot price-fixing round and the actual delivery hour of the concluded contracts is quite long (36 hours at the most). The Elbas market supplements the Elspot and the national Nordic real-time markets, as it allows a market player to adjust the market exposure between the DA auction on Elspot at noon and the actual hour of delivery the following day. Elbas is run by Nord Pool Finland Oy (formerly EL-EX Electricity Exchange Ltd.). It has been in operation since 1999. This market provides continuous power trading 24 hours a day covering individual hours in the same way as the Elspot market (one hour delivery period with $1 \mathrm{Mw}$ load). However, the contracts are open for trade only after the Elspot auction, so 24 new contracts are 


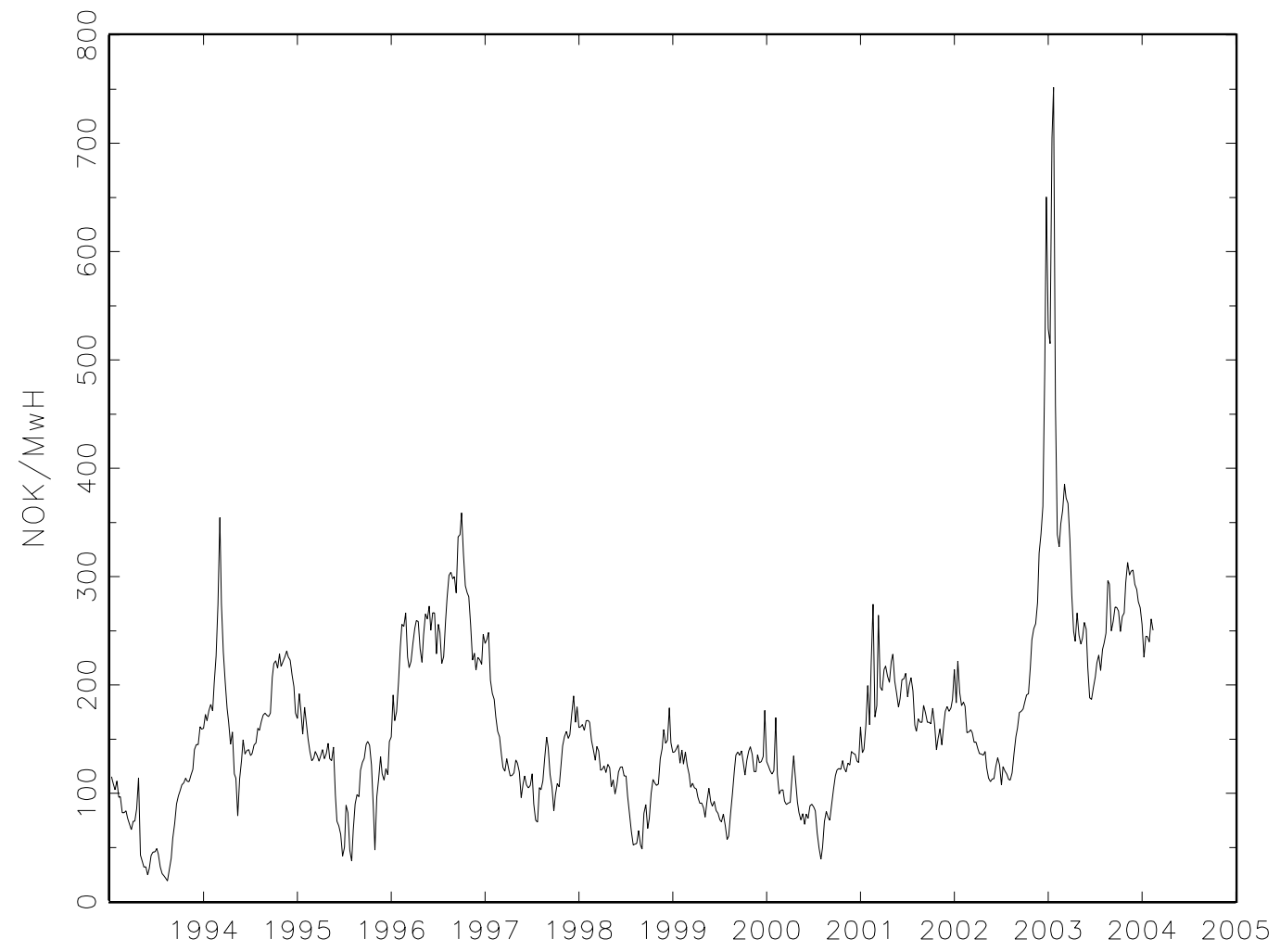

FiguRE 1. Time series of spot prices from Nord Pool in the period 1993-2004 (weekly average of day-ahead prices).

introduced daily. Just like Elspot, Elbas is a physical market for power trading in hourly contracts. The products can be traded up to one hour prior to delivery (two hours prior to delivery in Eastern Denmark). It only covers the trading areas of Finland, Sweden and Eastern Denmark, and the amount of power traded is limited by the free cross-border transmission capacity. Unlike the Elspot market, Elbas allows participants to buy and sell the same physical contract several times before delivery, and the position can be closed prior to delivery, so that no actual physical delivery is necessary.

2.2. Financial electricity contracts. Specifications and rules of trading for financial electricity contracts vary among the different power exchanges. The fact that these contracts are settled financially against a reference price, implies that the market place for financial electricity contracts does not require central coordination. They can be considered as side bets on the physical system. We will describe contracts traded on Nord Pool only. 
2.2.1. Swaps. Although contracts for future delivery of power are called futures or forwards, this denomination is not entirely correct. The basic exchange traded contracts at Nord Pool are written on the (weighted) average of the (hourly) system price over a specified delivery period (DP). During the delivery period the contract is settled in cash against the system price, hence, financial electricity contracts are in fact swap contracts, exchanging a floating spot price against a fixed price. We will therefore refer to these contracts as swaps in the rest of this paper. Furthermore, when we refer to the spot price in our mathematical model, this should be interpreted as whatever reference price a given exchange has tied its financial contracts to. The specified reference price is typically the day-ahead price described in the section above. In this way the financial electricity contracts are not the relevant risk management vehicles for hedging real-time electricity price risk. Contracts on Nord Pool are not traded during the delivery period, and market participants typically close their position prior to the delivery period. We consider only swap dynamics in the trading period in our mathematical model specification in the following sections. The time period that the contract is available for trading is called the trading period. The term "time to maturity" used for fixed maturity forward contracts is replaced time to delivery (TTD).

Nord Pool has facilitated trading in financial electricity contracts since 1995. In Table 1 we list the closing prices of swaps traded on Nord Pool March 25, 2004. The coloumn DP provides the length of the delivery period for each contract measured in days, and the two columns Start and End provide the start and end date of delivery. Since the contracts are settled against hourly day-ahead prices (the Nord Pool system price), and all contracts are base load contracts, the underlying amount of electrical energy is determined by

$$
D P \times 24 \times M w H
$$

To be able to compare contracts with different delivery periods, prices are listed in Norwegian Crowns (NOK) for $1 \mathrm{MWh}$ of energy delivered as a constant flow during the delivery period. Note that some contracts are listed in the European Currency (EUR). At the time of writing Nord Pool is in a transformation phase. Ultimately all contracts will be quoted in EUR. Since the start in 1995, contract specifications has changed several times. Peak load contracts were available the first couple of years, but they no longer trade due to low liquidity. There has also been a change of delivery periods for monthly and seasonal contracts. Block contracts with delivery periods of exactly 4 weeks, have been replaced by monthly contracts with delivery period equal to the respective calendar month. Quarterly seasonal contracts are replacing the former three-season regime. The quarterly contracts were first introduced for the year 2005.

In the first trading day in January each year, four new quarterly contracts are listed. The new Q1 contract trades for two years, the new Q2 contract trades for two years and three months etc. A new yearly contract that trades for 3 years is also introduced. This 
Ticker Currency Close Start End TTD DP

Panel A: Daily contracts

$\begin{array}{lllllll}\text { ENOD2603-04 } & \text { NOK } & 250.00 & 26.03 .04 & 26.03 .04 & 1 & 1 \\ \text { ENOD2703-04 } & \text { NOK } & 245.74 & 27.03 .04 & 27.03 .04 & 2 & 1 \\ \text { ENOD2803-04 } & \text { NOK } & 245.74 & 28.03 .04 & 28.03 .04 & 3 & 1\end{array}$

Panel B: Weekly contracts

$\begin{array}{llrrrrr}\text { ENOW14-04 } & \text { NOK } & 252.00 & 29.03 .04 & 04.04 .04 & 4 & 7 \\ \text { ENOW15-04 } & \text { NOK } & 244.00 & 05.04 .04 & 11.04 .04 & 11 & 7 \\ \text { ENOW16-04 } & \text { NOK } & 250.00 & 12.04 .04 & 18.04 .04 & 18 & 7 \\ \text { ENOW17-04 } & \text { NOK } & 251.00 & 19.04 .04 & 25.04 .04 & 25 & 7 \\ \text { ENOW18-04 } & \text { NOK } & 251.13 & 26.04 .04 & 02.05 .04 & 32 & 7 \\ \text { ENOW19-04 } & \text { NOK } & 246.13 & 03.05 .04 & 09.05 .04 & 39 & 7 \\ \text { ENOW20-04 } & \text { NOK } & 244.13 & 10.05 .04 & 16.05 .04 & 46 & 7 \\ \text { ENOW21-04 } & \text { NOK } & 235.00 & 17.05 .04 & 23.05 .04 & 53 & 7\end{array}$

Panel C: Monthly contracts

\begin{tabular}{lllllrr}
\hline ENOMAPR-04 & NOK & 249.50 & 01.04 .04 & 30.04 .04 & 7 & 30 \\
ENOMMAY-04 & NOK & 242.00 & 01.05 .04 & 31.05 .04 & 37 & 31 \\
ENOMJUN-04 & NOK & 234.00 & 01.06 .04 & 30.06 .04 & 68 & 30 \\
ENOMJUL-04 & NOK & 226.00 & 01.07 .04 & 31.07 .04 & 98 & 31 \\
ENOMAUG-04 & NOK & 243.75 & 01.08 .04 & 31.08 .04 & 129 & 31 \\
ENOMSEP-04 & NOK & 252.00 & 01.09 .04 & 30.09 .04 & 160 & 30
\end{tabular}

Panel D: Seasonal contracts

\begin{tabular}{llrrrrr}
\hline FWSO-04 & NOK & 239.75 & 01.05 .04 & 30.09 .04 & 37 & 153 \\
FWV2-04 & NOK & 263.10 & 01.10 .04 & 31.12 .04 & 190 & 92 \\
FWV1-05 & NOK & 268.50 & 01.01 .05 & 30.04 .05 & 282 & 120 \\
FWSO-05 & NOK & 206.25 & 01.05 .05 & 30.09 .05 & 402 & 153 \\
FWV2-05 & NOK & 237.63 & 01.10 .05 & 31.12 .05 & 555 & 92 \\
ENOQ1-06 & EUR & 31.15 & 01.01 .06 & 31.03 .06 & 647 & 90 \\
ENOQ2-06 & EUR & 25.25 & 01.04 .06 & 30.06 .06 & 737 & 91 \\
ENOQ3-06 & EUR & 24.30 & 01.07 .06 & 30.09 .06 & 828 & 92 \\
ENOQ4-06 & EUR & 28.38 & 01.10 .06 & 31.12 .06 & 920 & 92
\end{tabular}

Panel E: Yearly contracts

\begin{tabular}{llrrrrr}
\hline FWYR-05 & NOK & 234.25 & 01.01 .05 & 31.12 .05 & 282 & 365 \\
ENOYR-06 & EUR & 27.20 & 01.01 .06 & 31.12 .06 & 647 & 365 \\
ENOYR-07 & EUR & 27.50 & 01.01 .07 & 31.12 .07 & 1012 & 365
\end{tabular}

TABle 1. Closing prices on futures and forward closing prices from Nord Pool, March 25, 2004. TTD and DP are short-hand for "time to delivery" and "delivery period", respectively. Both are measured in days. 


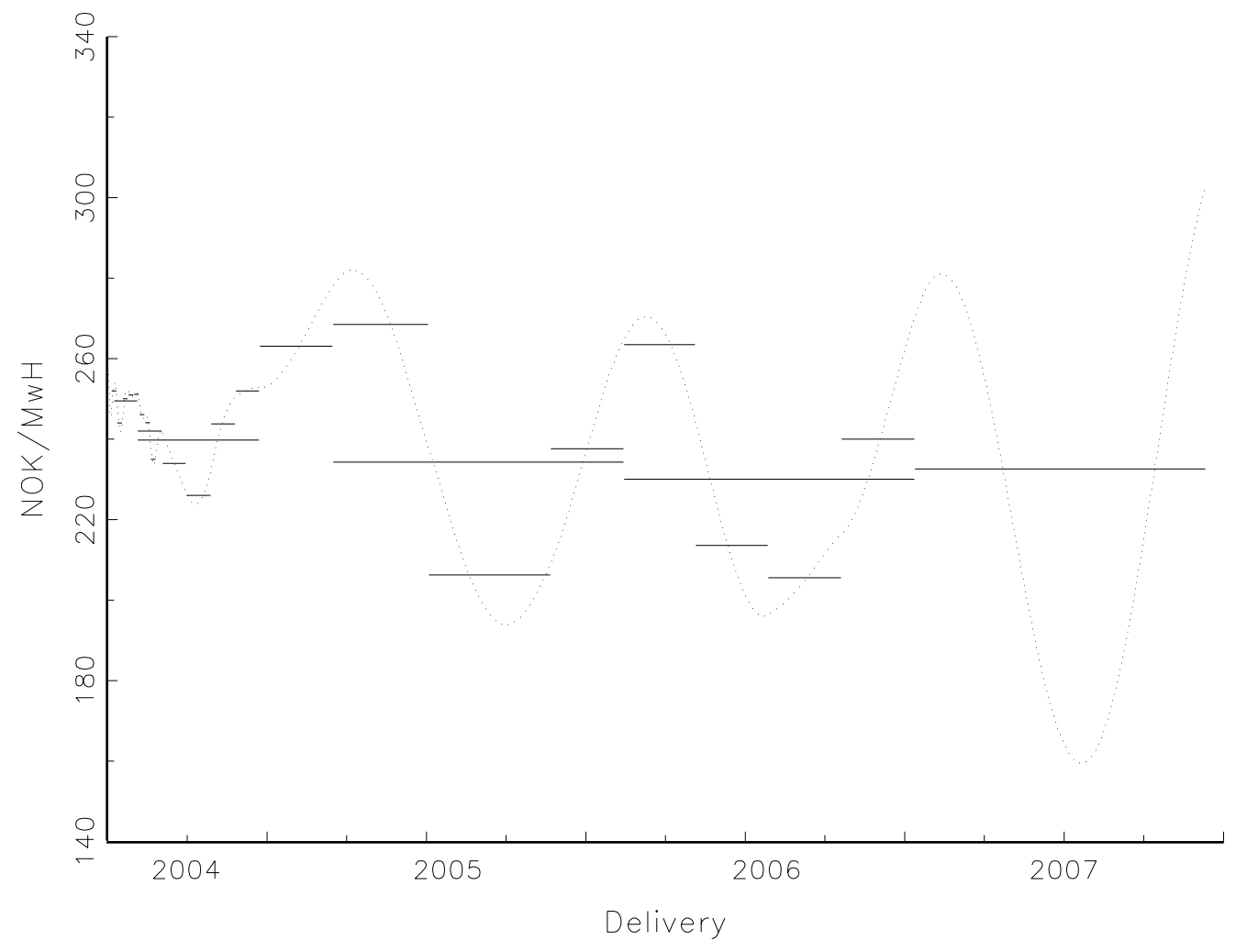

Figure 2. Electricity swap prices from Nord Pool and a corresponding smooth forward curve on March 25, 2005.

way it is possible to hedge the exposure to electricty prices in the Nordic market 3 to 4 years into the the future at all times. Every month a monthly contract is unlisted, and a new one is introduced that trades for 6 months. Each week one weekly contract is unlisted, and a new one is introduced that trades for 8 weeks. New daily contracts are introduced every Thursday. The Friday contract only trades for one day. All contracts trade until the last trading day prior to the delivery period.

The contracts differ when it comes to how settlement is carried out during the trading period. Daily and weekly contracts are futures-type contracts. The value of such a contract is calculated daily, reflecting changes in the contract's market prices. These changes are settled at each participant's margin account. We will use the term futures-style swaps for contracts that are marked-to-market in the trading period.

2.2.2. Swaptions and contracts for differences (CfDs). Nord Pool's financial market also includes option contracts and Contracts for Differences (CfD). The options are written on the the swap contracts (swaptions), and they are of European type. Exercise day is set as the third Thursday in the month before the delivery period of the underlying contract 
starts. Swaptions are traded on some seasonal and yearly contracts. Asian options written directly on the system price are frequently traded in the OTC market. As mentioned above, area prices may differ from the system price in case of congestion. CfDs are defined as the area price minus the system price. The different tradeable area prices are Oslo, Stockholm, Copenhagen, Aarhus and Helsinki.The CfDs are defined similar to the swap contracts with identical delivery periods. However shorter delivery periods than one month do not trade. Using CfDs in combination with swaps allows a market participant to effectively remove the price risk associated with congestion. ${ }^{4}$

2.3. A brief survey of existing literature. Bessembinder and Lemon [7] develop an equilibrium model for an electricity market. Their model allows for a finite number of players on the demand and supply side of the market, all with physical capabilities of either producing or consuming power. A discrete time equilibrium model for spot (RT) and forward (DA) markets are investigated, and the authors find that the risk premium in the forward market depends on both the price variation (standard deviation) and the right-skewedness of electricity spot prices. Longstaff and Wang [41] find empirical support for these propositions using data from the Pennsylvania, New Jersey and Maryland (PJM) electricity market. The model in [7] also predict decreasing forward premium if speculators are allowed to enter the market. Quite recently speculators were allowed to make DA bids in the PJM market. The bids from speculators are treated as side bets (speculator bids affect price but not capacity), since they can make no physical consumption in- or outtake to the system. Saravia [48] finds that the risk premium has indeed decreased after speculators are allowed to participate in the forward market.

Electricity contracts for physical delivery are mostly traded in auctions. Designing electricity auctions have been studied by a number og authors (see for instance [18] and references therein). Some efforts have been made in bridging the gap between auctions in the DA and/or RT market and financial electricity contracts. Typically the equilibrium spot price is modeled endogenously as the outcome from an auction, and then financial electricity futures prices can be derived (see for instance [52] and [27] for different approaches). Barlow [2] suggest a microeconomic based equilibrium diffusion model. Elliot et al. [17] model the spot price as a function of the number of power stations on line. In their model, the power stations going on/off line introduce jumps in the spot price process.

The time series properties of electricity prices have been studied quite intensively. Examples are Wolak [58], Knittel and Roberts [36], Escribano et al. [16], Leon og Rubia [40] and Guthrie and Videbeck [24]. Note that, as opposed to for instance Longstaff and Wang

\footnotetext{
${ }^{4}$ See http://www.nordpool.no/nordpool/financial/index.html for details on the different financial contracts traded on Nord Pool. In this paper our main focus is on the financial swap contracts. For those interested in pricing CfDs, see Kristiansen [39]. For a spot based approach to swaption pricing, see Hjalmarson [29].
} 
[41], this strand of literature refers to DA and RT prices as spot prices. In this literature it is common to define daily average DA prices as the spot price. Thus, varying prices during the day is smoothed away, and only weekly and yearly seasonality is left in the time series. The exceptions are the studies by Guthrie and Videbeck [24] and Knittel and Roberts [36]. The first study use half-hourly RT prices from the New Zealand electricity market, and they model each half hour separately. Knittel and Roberts [36] use hourly DA prices and stack the data into one long time series. The main findings from these studies are a strong degree of mean reversion in prices and occational jumps in addition to daily- weekly and yearly seasonality. These effects are present in all electricity markets, but vary somewhat from one regional market to the other.

All the studies mentioned in the above paragraph are discrete time models. A number of continuous time models have been suggested in the literature as well. The usual framework is an exogenous spot price dynamics. For a given assumption for the market price of risk, the spot dynamics under some equivalent martingale measure can be stated, and solutions to financial electricity contracts can be calculated. Lucia and Schwartz [42] suggest models (both one- and two factor models) that capture both mean reversion and seasonality. The models are estimated using spot price data (daily average of DA prices) in the Nordic market. the models are then calibrated to swap price data. Their model is close to the two-factor model suggested by Pilipovic [46], but the latter produces somewhat more complicated closed form expression for fixed delivery futures contracts. Deng [15] adds jumps to the spot price dynamics, and calibrates his models to American spot price data. Benth et al. [4] consider a general exponential multifactor mean-reversion model with jumps and calculate explicitly fixed-delivery forward prices from this. The model is calibrated to smoothed swap data from Nord Pool. Geman and Roncoroni [23] suggest an exponential mean-reversion model with state-dependent jumps. The model is able to capture both the spikes observed for electricity prices along with the distributional properties, and is tested empirically for many electricity markets. Benth, Kallsen and Meyer-Brandis [5] suggest an additive mean-reverting model with jumps, where the jumps are so that the spot prices remain positive. For this model one can derive explicit swap prices, and computable expressions for prices of options on swaps.

Some authors have suggested a more direct approach to the modeling of financial electricity contracts by setting up a model for fixed-delivery futures contracts in the framework of Heath-Jarrow-Morton. This approach was introduced to energy markets by Clewlow and Strickland [13]. Bjerksund et al. [9], Koekebakker and Ollmar [38] and Keppo et al. [35] have applied this model in the electricity market. They consider modeling a continuum of fixed delivery forward contracts, where the traded swap contracts become integrals of traditional forward contracts. Hence, to fix the model we need to produce a smooth curve of forward prices from swap prices. In Figure 2 we have computed a forward curve from the swap prices reported in Table 1 . The horizontal lines represent the delivery period and 
the price level of the swap prices from Table 1 along with a forward curve. In a model with a continuum of forward prices, the price of a swap contract is simply the integral of forward prices properly weighted. The curve is constructed in such a way that market prices on the traded swaps can be recaptured from the forward curve. The computation of the curve is based on a criterion called maximum smoothness suggested by Adams and van Deventer [1]. For details on how this approach can be applied to swap contracts we refer to Ollmar [45]. We note in passing that this is only one of many different ways to produce a forward curve from actual prices, and we refer to Fleten and Lemming [20] for an alternative approach.

Our studies are closely related to the abovementioned papers. However, instead of stating the dynamics of the fixed-delivery forward curve, we argue that it is a more convenient approach to specify the dynamics of traded swap contracts directly. Such a market model approach allows us to utilise the information in the traded swaps, without relying on some ad hoc smoothing algorithm. In the following Sections we investigate the mathematical relationship between spot-, fixed delivery forward- and swap price modelling.

\section{SOME BASIC NOTATION AND DEFINITIONS}

Assume $T<\infty$ and let $(\Omega, \mathcal{F}, Q)$ be a complete filtered probability space, with an increasing and right-continuous filtration $\left\{\mathcal{F}_{t}\right\}_{t \in[0, T]}$ where $\mathcal{F}_{0}$ contains all sets of probability zero in $\mathcal{F}{ }^{5}$ Note that we work directly under the risk-neutral probability $Q$. The physical (or market) probability measure is denoted $P$, being equivalent to $Q$. From time to time we will refer to this measure, but mostly we are concerned with modeling under the riskneutral measure $Q$, and choose to specify all process under this directly.

Denote by $W(t)$ a standard Brownian motion and $L(t)$ a square-integrable Lévy process being a martingale and independent of $W(t)$. We choose to work with the right-continuous version of $L$ with left-limits (the so-called cadlag version of $L(t)$ ). The Lévy-Kintchine representation of $L(t)$ is

$$
L(t)=\int_{0}^{t} \int_{\mathbb{R}} z \tilde{N}(d z, d t),
$$

where $N(d z, d t)$ is a homogeneous Poisson random measure with compensator $\ell(d z) d t$ and $\ell(d z)$ being the Lévy measure of $L(t)$ satisfying $\int_{\mathbb{R}} \min \left(1, z^{2}\right) \ell(d z)<\infty$. We use the notation $\widetilde{N}(d z, d t)$ for the compensated Poisson random measure, that is, $\widetilde{N}(d z, d t)=$ $N(d z, d t)-\ell(d z) d t$.

We suppose that our market consists of swap contracts with different delivery periods and a bond that yields a constant risk-free (continuously compounded) rate of return $r>0$. The contracts are base load contracts. Consider a swap contract with delivery period

\footnotetext{
${ }^{5}$ E.g, the filtered probability space satisfies the usual hypotheses, see Karatzas and Shreve [33]
} 
$\left[T_{1}, T_{2}\right]$, for $0<T_{1}<T_{2} \leq T$, and denote its price at time $0 \leq t \leq T_{1}$ by $F\left(t, T_{1}, T_{2}\right)$. Suppose that the price dynamics for $0 \leq t \leq T_{1} \leq T_{2} \leq T$ has the differential form

$$
d F\left(t, T_{1}, T_{2}\right)=\Sigma\left(t, T_{1}, T_{2}\right) d W(t)+\int_{\mathbb{R}} \Psi\left(t, T_{1}, T_{2}, z\right) \widetilde{N}(d z, d t)
$$

We assume $\Sigma$ and $\Psi$ to be sufficiently regular random fields such that the swap dynamics becomes a square-integrable martingale (see the sufficient Condition (SW) in Appendix A). Note that since no initial payment is made when entering a swap contract, its dynamics must be a martingale under the risk-neutral probability $Q$.

We shall consider cases where the market trades in forward (or futures, which have the same price dynamics when the risk-free return is constant, as it is assumed in our case) with fixed time of maturity as well. In this case we denote by $f(t, u)$ the price at time $t$ of a forward contract with delivery at time $0 \leq t \leq u \leq T$. Let the forward dynamics $f(t, u)$ for $0 \leq t \leq u \leq T$ be

$$
d f(t, u)=\sigma(t, u) d W(t)+\int_{\mathbb{R}} \psi(t, u, z) \widetilde{N}(d z, d t),
$$

where $\sigma$ and $\psi$ satisfy suitable conditions (see the sufficient Condition (F) in Appendix A) turning $f(t, u)$ into a square-integrable martingale. Note that the forward price is also a martingale under the risk-neutral probability $Q$ since the initial investment in such a contract is zero and therefore can not have any drift. Recall that the electricity markets do not trade forwards with fixed delivery time.

We also consider a spot price dynamics. Assume that the spot price at time $t$ is $S(t)$, with dynamics

$$
d S(t)=\alpha(t) d t+\beta(t) d W(t)+\int_{\mathbb{R}} \gamma(t, z) \widetilde{N}(d z, d t) .
$$

Suitable conditions on $\alpha, \beta$ and $\gamma$ such that the stochastic process $S$ becomes a squareintegrable semi-martingale are assumed (see Condition (S) in Appendix A).

As we have already mentioned, forward contracts with fixed delivery time does not exist in the electricity market. However, they are in our context theoretically meaningful as limiting versions of swaps when the delivery period becomes infinitesimally small, as we will see later. The spot price must be thought of as a real time electricity contracts with both time to maturity and delivery period approaching zero. In the mathematical model spot prices are fixed delivery forwards with zero time to maturity, an object that is hard to interpret directly in the electricity market. Both the day-ahead and real time markets are not perfectly fitting into this interpretation. In what to come we shall consider the hourly quoted system prices in the DA market as discrete samples from an underlying continuous-time spot price. 


\section{Forward MODELING USING THE HJM-APPROACH}

In this Section we start out with a dynamical model of the forward curve in order to derive a model for the swap price dynamics. That is, we assume that the market trades in forward contracts, and from arbitrage arguments we establish the link to swap contracts. Bjerksund et al. [9] propose this approach using a lognormal class of forward models. In view of the rich literature of HJM-modeling of forward rates in fixed-income markets (see e.g. Musiela and Rutkowski [44], and the references therein), this seems to be a rather natural starting point. However, as we shall see, the implied swap dynamics becomes very complicated, even for quite simple multiplicative models, and the analytical tractability for option pricing and VaR calculations that one may have had for the forward dynamics is lost. We derive the explicit swap dynamics, and discuss different models and their properties in this context.

Assume that the forward dynamics is given by (3.2). Appealing to arbitrage arguments, we find that a swap contract with delivery in the period $\left[T_{1}, T_{2}\right]$ must be related to a continuum of forward contracts as

$$
F\left(t, T_{1}, T_{2}\right)=\int_{T_{1}}^{T_{2}} \widehat{w}\left(u ; T_{1}, T_{2}\right) f(t, u) d u .
$$

Here, we use the notation

$$
\widehat{w}(u ; s, t):=\frac{w(u)}{\int_{s}^{t} w(v) d v},
$$

for $0 \leq u \leq s<t$, where the function $w(u)$ is equal to 1 if the swap settles at maturity, while it is $w(u)=\exp (-r u)$ if the settlement takes place continuously during the delivery period. The following result is straightforward (see Appendix B for an argument):

Lemma 4.1. For $0 \leq t \leq T_{1}$, it holds that

$$
F\left(t, T_{1}, T_{1}\right):=\lim _{T_{2} \downarrow T_{1}} F\left(t, T_{1}, T_{2}\right)=f\left(t, T_{1}\right) \text {, a.e.t } \in\left[0, T_{1}\right] .
$$

Hence, a forward is a swap with zero delivery period. Note that we have imposed rather general conditions on the dynamics of $F$ and $f$, which gives this relation in an almost everywhere sense with respect to time $t$. With stronger conditions on the parameters in the respective dynamics, like e.g. lognormal models with continuous and bounded volatility function, the connection will hold for every $t$.

Our next Lemma shows that if the market trades in forward contracts the swap contracts will satisfy a natural no-arbitrage condition (see Appendix B for a proof):

Lemma 4.2. Consider a swap with delivery over the period $\left[T_{1}, T_{N}\right]$ and $N$ swaps with delivery over the disjoint periods $\left[T_{i}, T_{i+1}\right], i=1, \ldots, N-1$ and $T_{i}<T_{i+1}$, where the union 
of these intervals coincides with $\left[T_{1}, T_{N}\right]$. Then the following holds

$$
F\left(t, T_{1}, T_{N}\right)=\sum_{i=1}^{N-1} w_{i} F\left(t, T_{i}, T_{i+1}\right)
$$

where $w_{i}=\int_{T_{i}}^{T_{i+1}} w(u) d u / \int_{T_{1}}^{T_{N}} w(u) d u$, for $i=1,2, \ldots, N-1$.

Note that the relation (4.1) can be considered as a continuous version of (4.4): Choose $T_{i}=T_{1}+(i-1) \cdot \Delta, i=1, . ., N$ and $\Delta=\left(T_{N}-T_{1}\right) / N$ in (4.4). After letting $N \rightarrow \infty$ and appealing to Lemma 4.1, we reach (4.1) .

4.1. The implied swap dynamics. We now proceed with deriving the coefficient functions of the swap dynamics (3.1), assuming that the forward dynamics (3.2) is given. In order to do so, we need to impose some additional measurability and integrability conditions on the coefficient functions in the respective dynamics (3.1) and (3.2). These conditions are natural in view of the assumption in the stochastic Fubini Theorem (see Protter [47, Thm. 46]). However, we have stated them in Appendix A due to their rather technical character. The proof of the following theorem can be found in Appendix B.

Theorem 4.3. Assume Condition ( $\boldsymbol{F}-\boldsymbol{S} \boldsymbol{W})$ (see Appendix A) holds for the coefficients of the forward dynamics (3.2). Then the coefficient functions in the forward dynamics are related to the swap dynamics in the following way:

$$
\begin{aligned}
\Sigma\left(t, T_{1}, T_{2}\right) & =\int_{T_{1}}^{T_{2}} \widehat{w}\left(u ; T_{1}, T_{2}\right) \sigma(t, u) d u, \\
\Psi\left(t, T_{1}, T_{2}, z\right) & =\int_{T_{1}}^{T_{2}} \widehat{w}\left(u ; T_{1}, T_{2}\right) \psi(t, u, z) d u .
\end{aligned}
$$

Following the arguments for Lemma 4.1, it immediately follows from this Theorem that the $\operatorname{limits} \lim _{T_{2} \downarrow T_{1}} \Sigma\left(t, T_{1}, T_{2}\right)$ and $\lim _{T_{2} \downarrow T_{1}} \psi\left(t, T_{1}, T_{2}, z\right)$ exist for almost every $0 \leq t \leq T_{1}$, and moreover, that

$$
\begin{aligned}
\sigma\left(t, T_{1}\right) & =\lim _{T_{2} \downarrow T_{1}} \Sigma\left(t, T_{1}, T_{2}\right):=\Sigma\left(t, T_{1}, T_{1}\right), \text { a.e. } t \in\left[0, T_{1}\right] \\
\psi\left(t, T_{1}, z\right) & =\lim _{T_{2} \downarrow T_{1}} \psi\left(t, T_{1}, T_{2}, z\right):=\Psi\left(t, T_{1}, T_{1}, z\right), \text { a.e. } t \in\left[0, T_{1}\right] .
\end{aligned}
$$

The standard choice of a forward model in energy markets is the lognormal, or geometric Brownian motion dynamics (see e.g. Clewlow and Strickland [13]). An extension of this to the jump diffusion case could be

$$
d f(t, u)=\bar{\sigma}(t, u) f(t, u) d W(t)+\int_{\mathbb{R}} \bar{\psi}(t, u, z) f(t-, u) \tilde{N}(d t, d u) .
$$

Note that since we have assumed that $L(t)$ is right-continuous, the solution of the stochastic differential equation (4.7) will become right-continuous. In order to have a predictable 
integrand when we integrate with respect to the compensated Poisson random measure $\widetilde{N}$, we must use the left-limit of $f$, namely $f(t-, u)$. We assume that $\bar{\sigma}$ and $\bar{\psi}$ are continuous and bounded deterministic functions, being differentiable with respect to $u$ with bounded derivatives. Furthermore, to assure that the forward price is positive, we suppose that $\bar{\psi}(t, u, z)>-1$. From (4.5), the volatility structure of the swap price dynamics becomes

$$
\Sigma\left(t, T_{1}, T_{2}\right)=\int_{T_{1}}^{T_{2}} \widehat{w}\left(u ; T_{1}, T_{2}\right) \bar{\sigma}(t, u) f(t, u) d u,
$$

which yields, after an integration-by-parts

$$
\Sigma\left(t, T_{1}, T_{2}\right)=\bar{\sigma}\left(t, T_{2}\right) F\left(t, T_{1}, T_{2}\right)-\int_{T_{1}}^{T_{2}} \partial_{2} \bar{\sigma}(t, u) \int_{T_{1}}^{u} \widehat{w}\left(\tau ; T_{1}, T_{2}\right) f(t, \tau) d \tau d u .
$$

Here, $\partial_{2}$ denotes partial differentiation with respect to the second variable of the function in question. Now, observing that $\widehat{w}\left(\tau ; T_{1}, T_{2}\right) / \widehat{w}\left(\tau ; T_{1}, u\right)$ is independent of $\tau$, we obtain

$$
\Sigma\left(t, T_{1}, T_{2}\right)=\bar{\sigma}\left(t, T_{2}\right) F\left(t, T_{1}, T_{2}\right)-\int_{T_{1}}^{T_{2}} \partial_{2} \bar{\sigma}(t, u) \frac{\widehat{w}\left(\tau ; T_{1}, T_{2}\right)}{\widehat{w}\left(\tau ; T_{1}, u\right)} F\left(t, T_{1}, u\right) d u .
$$

Similarily, we find

$$
\Psi\left(t, T_{1}, T_{2}, z\right)=\bar{\psi}\left(t, T_{2}, z\right) F\left(t, T_{1}, T_{2}\right)-\int_{T_{1}}^{T_{2}} \partial_{2} \bar{\psi}(t, u, z) \frac{\widehat{w}\left(\tau ; T_{1}, T_{2}\right)}{\widehat{w}\left(\tau ; T_{1}, u\right)} F\left(t, T_{1}, u\right) d u
$$

As we can see, the swap dynamics does not preserve the multiplicative structure of the forward dynamics. In fact, the dynamics of the swap contract with settlement over the period $\left[T_{1}, T_{2}\right]$ fails even to be a Markov process since it depends on the dynamics of all other swap contracts with settlement over the period $\left[T_{1}, u\right]$, for every $T_{1}<u \leq T_{2}$. We remark that the swap dynamics can be interpreted as an infinite dimensional stochastic process, and in this sense it will be multiplicative and therefore Markovian (see DaPrato and Zabzcyk [14]).

Consider now the special case of a lognormal forward dynamics

$$
d f(t, u)=\bar{\sigma}(t, u) f(t, u) d W(t) .
$$

The implied dynamics for a swap contract with delivery over $\left[T_{1}, T_{2}\right]$ has a volatility structure given by (4.8), that is, the dynamics is

$$
\begin{aligned}
d F\left(t, T_{1}, T_{2}\right)= & \bar{\sigma}\left(t, T_{2}\right) F\left(t, T_{1}, T_{2}\right) d W(t) \\
& -\int_{T_{1}}^{T_{2}} \partial_{2} \bar{\sigma}(t, u) \frac{\widehat{w}\left(\tau ; T_{1}, T_{2}\right)}{\widehat{w}\left(\tau ; T_{1}, u\right)} F\left(t, T_{1}, u\right) d u d W(t) .
\end{aligned}
$$

We see that when $\bar{\sigma}$ is not a function of the expiration date of the forward, that is $\partial_{2} \bar{\sigma}(t, u)=$ 0 and thus $\bar{\sigma}(t, u)=\bar{\sigma}(t)$, we obtain a lognormal swap dynamics:

$$
d F\left(t, T_{1}, T_{2}\right)=\bar{\sigma}(t) F\left(t, T_{1}, T_{2}\right) d W(t) .
$$


This is in fact the only case where lognormality of the forward dynamics is carried over to the swap dynamics. Using a volatility which does not depend on time to maturity in the forward specification is known as the Black-76 model, and first introduced in Black [8]. We see that the modeling could easily be reversed, that is, we could first start out with a Black-76 specification of the swap dynamics as in (4.11), and from this we would get a forward dynamics given by

$$
d f(t, u)=\bar{\sigma}(t) f(t, u) d W(t),
$$

which is the Black-76 forward dynamics. We remark in passing that a popular choice of $\bar{\sigma}(t)$ is to assume it constant. This is indeed the choice of Black [8].

In realistic models of the volatility, $\bar{\sigma}(t, u)$ depends strongly on the time of delivery of the forward. Hence, we do not have a swap dynamics which is lognormal, but instead given by (4.10). We know that this dynamics stems from a weighted integral of forwards (4.1), which in this situation will be a weighted integral of geometric Brownian motions. Therefore, we are led to interpret plain vanilla call options on swap contracts as average-type options. Although a bit simpler than Asian options, there exists no analytic pricing formula for such options, and numerical procedures are called for. This makes pricing a rather difficult task, and the same concerns hold for risk management (or VaR-calculations). Option pricing of forward contracts will be discussed in more detail in Section 7.

To overcome this problem, Bjerksund et al. [9] suggest to approximate the implied swap dynamics (4.10) with a lognormal model where the volatility function is given by $\int_{T_{1}}^{T_{2}} \widehat{w}\left(u ; T_{1}, T_{2}\right) \bar{\sigma}(t, u) d u$, that is, they do the approximation

$$
\bar{\sigma}\left(t, T_{2}\right)-\int_{T_{1}}^{T_{2}} \partial_{2} \bar{\sigma}(t, u) \frac{\widehat{w}\left(\tau ; T_{1}, T_{2}\right)}{\widehat{w}\left(\tau ; T_{1}, u\right)} \frac{F\left(t, T_{1}, u\right)}{F\left(t, T_{1}, T_{2}\right)} d u \approx \int_{T_{1}}^{T_{2}} \widehat{w}\left(u ; T_{1}, T_{2}\right) \bar{\sigma}(t, u) d u .
$$

With the explicit knowledge of the true volatility structure of the swap dynamics in (4.8), one can evaluate the reasonability of such an approximation. First of all, the returns of the swap following the approximation of Bjerksund et al. [9] will be independent and normally distributed. The independence is not valid for the correct swap dynamics, being

$$
\frac{d F\left(t, T_{1}, T_{2}\right)}{F\left(t, T_{1}, T_{2}\right)}=\bar{\sigma}\left(t, T_{2}\right) d W(t)-\int_{T_{1}}^{T_{2}} \partial_{2} \bar{\sigma}(t, u) \frac{\widehat{w}\left(\tau ; T_{1}, T_{2}\right)}{\widehat{w}\left(\tau ; T_{1}, u\right)} \frac{F\left(t, T_{1}, u\right)}{F\left(t, T_{1}, T_{2}\right)} d u d W(t) .
$$

Hence, the swap returns will depend on the current state of the swap prices, which are not independent over time increments. Even though we may have that the approximated volatility matches the standard deviation of the returns in the correct swap model, the time dependence will be wrongly modelled. This may have influence in evaluation of path dependent options on swaps or on risk management.

Let us discuss some issues concerning estimation of these swap models to market data. Starting out with a forward dynamics, we have essentially two ways to fit the swap model to quoted market prices: Either we can estimate the implied swap dynamics, or we can 
estimate the forward dynamics. The former is very hard since the implied swap dynamics becomes rather complicated for natural choices of the volatility structure of the forward. The latter, on the other hand, is theoretically impossible since the electricity market does not trade in forwards with fixed delivery time. There exists no such data. However, following Ollmar [45] one may create a history of forward curves from the swap price data using a smoothing procedure. This procedure goes in two steps, where the first is to transform swap prices into prices for artificial forward contracts. The next step is to smooth such prices to make a forward curve, which then is used for estimating the forward dynamics. An example of this procedure applied to electricity data was presented in Figure 2.

Another problem we encounter when fitting such models to data is that the dynamics of the forward is specified under the risk-neutral probability measure $Q$. When modeling the forward dynamics, this seems to be the preferred approach in fixed-income markets (following the HJM-approach). As a consequence, the swap dynamics is a martingale under the risk-neutral measure as well. Fitting the forward model to data, however, leads to some practical problems. The data are observed under the physical (or objective) probability $P$ and not under $Q$. Since $Q$ is equivalent to $P$ by definition, we can appeal to the Girsanov Theorem (see e.g. Shiryaev [51]) for finding the dynamics of the forward (and the swap) under $P$ from (3.2) (and (3.1) ). An additional drift term will enter the dynamics, which can be interpreted as a market price of risk, measuring the "premium" charged by the market for not being able to hedge the forward using the spot. Denoting this random field by $\lambda(t, u)$, we have that the forward dynamics under $P$ can be stated as (we assume that $\lambda$ is sufficiently regular to preserve the square-integrability of $f$ )

$$
d f(t, u)=\lambda(t, u) d t+\sigma(t, u) d B(t)+\int_{\mathbb{R}} \psi(t, u, z) \tilde{N}(d z, d t)
$$

where $B$ is a Brownian motion under $P$. Note that $\widetilde{N}$ is the compensated Poisson random measure for the process $L$, which we assumed to be a Lévy process under $Q$. A similar calculation as in Theorem 4.3 (assuming sufficient integrability conditions on $\lambda$ ), we find that the swap dynamics becomes

$$
d F\left(t, T_{1}, T_{2}\right)=\Lambda\left(t, T_{1}, T_{2}\right) d t+\Sigma\left(t, T_{1}, T_{2}\right) d B(t)+\int_{\mathbb{R}} \Psi\left(t, T_{1}, T_{2}\right) \widetilde{N}(d z, d t)
$$

where

$$
\Lambda\left(t, T_{1}, T_{2}\right)=\int_{T_{1}}^{T_{2}} \widehat{w}\left(u ; T_{1}, T_{2}\right) \lambda(t, u) d u .
$$

Assigning a model for the market price of risk enables us to estimate the forward dynamics to data, at least in the lognormal case. But when we include Lévy noise in the dynamics, some additional statistical issues are raised. In general, a Lévy process under $Q$ 
is not necessarily a Lévy process under $P$ (and vice versa). Indeed, the Girsanov Theorem can map a Lévy process into a rather general semimartingale process. Thus, nice probabilistic features specified under one probability can get completely lost after changing to the other probability leading to statistical problems when fitting the actual model to data. However, if we do a change-of-measure which only transforms the Brownian part of the dynamics, we will preserve the Lévy property of $L$ (see e.g. Benth et. al [4]), and even the class of infinitly divisible distributions generating the marginals of the Lévy process. In general, however, a change of probability measure affects the distributional properties of the process $L$ and may transform it into a process being difficult to handle from a statistical point of view. One may argue that in this case it is better to model under $P$ (and thus assume $L$ to be a Lévy process under $P$ instead). Although this may look tempting, we run into exactly the same problems when calculating prices of options written on the swap. To find option prices, we must introduce a risk-neutral probability, which means that the Lévy property of $L$ can be lost, and pricing may become difficult.

We note that all the theoretical considerations on the forward and swap dynamics can easily be extended to include several Brownian motions and Lévy processes. For modeling purposes, it may be more realistic to use a multidimensional noise term for the forward and swap dynamics, see Keppo et al. [35] and Koekebakker and Ollmar [38]. However, for simplicity we have chosen to restrict our attention to only one Wiener and jump term in the pricing dynamics.

4.2. The implied spot dynamics. We now move on to study the relation with a spot dynamics, and we suppose that the spot price follows (3.3).

Appealing to the general arbitrage theory, the forward price at time $t$ is linked to the spot via the conditional expectation of the spot at delivery time $u \geq t$ (see e.g. Musiela and Rutkowski [44]),

$$
f(t, u)=\mathbb{E}_{Q}\left[S(u) \mid \mathcal{F}_{t}\right]
$$

which yields the well-known relation

$$
S(t)=f(t, t)
$$

since the spot price is an adapted process. We will use the relations (4.1) and (4.16) to establish explicit connections between the dynamics of the different contracts.

First, we establish an explicit connection between the coefficient functions of the forward and spot dynamics. The following result is a slight generalization of Prop. 13.1.1 in Musiela and Rutkowski [44], where they derive an analogous result for the connection between forward and short rates in fixed-income markets, however, for stochastic processes without jumps. Appealing to the Fubini Theorem in Protter [47], we find (see Appendix B for a proof) 
Theorem 4.4. Assume Condition (F-S) (see Appendix A) holds for the coefficients of the forward dynamics (3.2). Then the spot dynamics (3.3) becomes

$$
d S(t)=\alpha(t) d t+\sigma(t, t) d W(t)+\int_{\mathbb{R}} \psi(t, t, z) \widetilde{N}(d z, d t),
$$

where

$$
\alpha(t)=\partial_{2} f(0, t)+\int_{0}^{t} \partial_{2} \sigma(s, t) d W(s)+\int_{0}^{t} \int_{\mathbb{R}} \partial_{2} \psi(s, t, z) \widetilde{N}(d z, d s) .
$$

Recall that the notation $\partial_{2}$ means differentiation with respect to the second argument.

Consider again the forward dynamics in (4.7). We find that

$$
\sigma(t, t)=\bar{\sigma}(t, t) S(t), \quad \psi(t, t, z)=\bar{\psi}(t, t, z) S(t-),
$$

which implies that the spot dynamics preserves the multiplicative structure in the diffusive terms of the forward dynamics. The drift, however, will not be multiplicative in general, in fact not even Markovian, and we are thus led to a spot dynamics which is not a lognormal process when $\bar{\psi}=0$. Note that if the spot could be perfectly traded (like the stock market), the drift would be equal to $\alpha(t)=r S(t)$, since in this case the expected rate of return would be the risk-free one under the equivalent martingale measure. This would give a lognormal dynamics. It is the non-tradeability (or non-storability) of the spot which makes it fail to preserve the lognormal structure of the forward dynamics. Another example where the spot dynamics becomes multiplicative (however, not Markovian), is when the volatility of the forward is independent of the time of maturity. Letting $\bar{\sigma}(t, u) \equiv \bar{\sigma}(t)$ and $\bar{\psi}(t, u, z) \equiv \bar{\psi}(t, z)$, we find

$$
\alpha(t)=\partial_{2} f(0, t)+\int_{0}^{t} \bar{\sigma}(s) \partial_{2} f(s, t) d W(s)+\int_{0}^{t} \int_{\mathbb{R}} \bar{\psi}(s, z) \partial_{2} f(s, t) \tilde{N}(d z, d s) .
$$

Now, observing that $\partial_{2} f(s, t)=\partial_{2} f(0, t) \cdot f(s, t) / f(0, t)$, we find

$$
\begin{aligned}
\alpha(t) & =\partial_{2} f(0, t)+\partial_{2} \ln f(0, t) \int_{0}^{t} \bar{\sigma}(s) f(s, t) d W(s)+\partial_{2} \ln f(0, t) \int_{0}^{t} \int_{\mathbb{R}} \bar{\psi}(s, z) f(s, t) \widetilde{N}(d z, d s) \\
& =\partial_{2} f(0, t)+\partial_{2} \ln f(0, t)(f(t, t)-f(0, t)) \\
& =\left\{\partial_{2} \ln f(0, t)\right\} S(t) .
\end{aligned}
$$

Hence, the spot dynamics is multiplicative (and lognormal for $\bar{\psi}=0$ ), with time-varying drift given by the logarithmic derivative of the initial forward curve with respect to time of maturity.

The connection between the swap and spot dynamics follows as a corollary to the Theorems 4.4 and 4.3 : 
Corollary 4.5. Suppose Conditions $(\boldsymbol{F}-\boldsymbol{S} \boldsymbol{W})$ and $(\boldsymbol{F}-\boldsymbol{S})$ hold (see Appendix A). Then the spot dynamics (3.3) becomes

$$
d S(t)=\alpha(t) d t+\Sigma(t, t, t) d W(t)+\int_{\mathbb{R}} \Psi(t, t, t, z) \widetilde{N}(d z, d t)
$$

where

$$
\alpha(t)=\frac{\partial}{\partial t} F(0, t, t)+\int_{0}^{t} \frac{\partial}{\partial t} \Sigma(s, t, t) d W(s)+\int_{0}^{t} \int_{\mathbb{R}} \frac{\partial}{\partial t} \Psi(s, t, t, z) \tilde{N}(d z, d s) .
$$

If we choose to model the different dynamics under the physical (or objective) probability $P$, we have to introduce a drift term in both the swap and forward dynamics. Following the same line of argument as for the volatility coefficients $\Sigma, \sigma, \Psi$ and $\psi$, it is straightforward to link the two drift coefficient functions under conditions that validate the application of the Fubini Theorem (non-stochastic). The drift of the spot dynamics will be correspondingly modified.

\section{Swap modeling Using the HJM-Approach: A general Model}

As we have discussed in Section 2, there is no trade in fixed delivery forwards or futures contracts for electricity. Hence, in the perspective of the HJM-approach, it is tempting to model the swap directly instead of going via the (non-existing) forwards, as discussed above. This approach will have the advantage that we can use the swap prices quoted in the market when calibrating the model, rather than a filtered version of them. A problem, however, is encountered with adopting the HJM-approach to modeling the swap dynamics: In the HJM-approach for forwards/futures, a dynamics is specified for all delivery times. It is supposed that the market trades in forward/futures with delivery at all times between today and some specified time horizon. Any martingale specification of this dynamics in the risk-neutral setting, will yield a dynamics which are arbitrage-free in the sense that one cannot create arbitrage by trading in forwards having different times to maturity (see e.g. Musiela and Rutkowski [44]). In the context of swaps, the no-arbitrage requirement becomes a bit more involved, since swaps deliver energy over a period, and not at some future fixed point in time.

A natural adoption of the HJM-framework, is to specify an arbitrage-free swap dynamics which is valid for all delivery periods within a pre-determined time horizon. We shall see that this goal is in practice very hard to achieve if we in addition want flexible models being easily tractable for risk management and option pricing. In this Section we analyse these modeling issues in more detail.

Assume the swap price $F\left(t, T_{1}, T_{2}\right)$ at time $t$ with delivery period $\left[T_{1}, T_{2}\right], t \leq T_{1}$, is modeled by the dynamics given in (3.1), where Condition (SW) in Appendix A holds to ensure square-integrability of the martingale process. In addition to this specification, we must assume that the two random fields $\Sigma$ and $\Psi$ are such that a no-arbitrage condition 
holds. Swap contracts with overlapping delivery periods must have prices being consistent with each other in order to avoid arbitrage opportunities: Consider a swap with delivery over the period $\left[T_{1}, T_{N}\right]$ and $N$ swaps with delivery over the disjoint periods $\left[T_{i}, T_{i+1}\right], i=$ $1, \ldots, N-1$. Then the following no-arbitrage condition must hold

$$
F\left(t, T_{1}, T_{N}\right)=\sum_{i=1}^{N-1} w_{i} F\left(t, T_{i}, T_{i+1}\right)
$$

where $w_{i}=\int_{T_{i}}^{T_{i+1}} w(u) d u / \int_{T_{1}}^{T_{N}} w(u) d u$, for $i=1,2, \ldots, N-1$. The condition (5.1) must hold for all swaps traded in the market where the settlement period can be decomposed into swaps with smaller disjoint delivery periods. If, on the other hand, the delivery period between two swaps are only partly overlapping, there is no such condition since one can not construct an arbitrage portfolio with the two contracts.

There are different interpretations of Condition (5.1). From a market point of view, it must hold for all the different swaps actually traded. That is, if the market for instance is trading in 4 swaps with delivery periods each quarter of the next year, and at the same time in a swap with delivery over the whole of next year, the no-arbitrage condition must hold for these 5 products. Taking the extreme point of view that the market trades in swaps for all possible delivery periods, Condition (5.1) becomes

$$
F\left(t, T_{1}, T_{2}\right)=\int_{T_{1}}^{T_{2}} \widehat{w}\left(u ; T_{1}, T_{2}\right) F(t, u, u) d u,
$$

for all $T_{1} \leq T_{2}$, where $0 \leq t \leq T_{1}$. This can be seen by choosing a homogeneous partition of the delivery period $\left[T_{1}, T_{2}\right]$, and letting this partition go to zero. Obviously, $F(t, u, u)$ will be the price of a forward at time $t$ with delivery at time $u \geq t$. In this Section we analyze the modeling of swaps using the extreme view of the HJM-approach where a dynamics for all delivery periods are to be presented. Hence, we must assume that condition (5.2) holds for our dynamical swap price model in order to avoid arbitrage.

The relation (5.2) is equivalent to structural conditions on the parameter functions $\Sigma$ and $\Psi$ :

$$
\begin{aligned}
\Sigma\left(t, T_{1}, T_{2}\right) & =\int_{T_{1}}^{T_{2}} \widehat{w}\left(u ; T_{1}, T_{2}\right) \Sigma(t, u, u) d u \\
\Psi\left(t, T_{1}, T_{2}, z\right) & =\int_{T_{1}}^{T_{2}} \widehat{w}\left(u ; T_{1}, T_{2}\right) \Psi(t, u, u, z) d u .
\end{aligned}
$$

We derive these relations using the stochastic Fubini theorem as in the proof of Theorem 4.3. To validate these calculations, we assume that the "forward"-parameter functions $\sigma(t, T):=\Sigma(t, T, T)$ and $\psi(t, T, z):=\Psi(t, T, T, z)$ satisfy conditions $(\mathbf{F})$ and $(\mathbf{F}-\mathbf{S W})$ in Appendix A. 
It is not straightforward to specify models for the swap dynamics which fullfill the no-arbitrage condition (5.2) (or, equivalently, the structural properties (5.3) -(5.4)). A natural class of models is the lognormal dynamics, which we now analyse in view of the noarbitrage condition (5.2). Let $\bar{\Sigma}\left(t, T_{1}, T_{2}\right)$ be a continuously differentiable (in all arguments) and bounded deterministic function describing the volatility, and consider the dynamics

$$
d F\left(t, T_{1}, T_{2}\right)=\bar{\Sigma}\left(t, T_{1}, T_{2}\right) F\left(t, T_{1}, T_{2}\right) d W(t) .
$$

From the discussion in Section 4, we know that if $\bar{\Sigma}$ does not depend on $T_{1}$ and $T_{2}$, that is, if $\bar{\Sigma}\left(t, T_{1}, T_{2}\right) \equiv \bar{\sigma}(t)$, the swap dynamics can be associated with a forward dynamics given by

$$
d f(t, u)=\bar{\sigma}(t) f(t, u) d W(t)
$$

Hence, in this case the no-arbitrage condition (5.2) will hold. We now argue that this Black76 model is the only lognormal swap dynamics specification which satisfy (5.2). We restrict our attention to swaps where $w(u)=1$, that is with settlement at maturity. Without loss of generality in the arguments to come, we assume that the initial swap curve is given by $F\left(0, T_{1}, T_{2}\right)=1$. An explicit representation of the swap price $F\left(t, T_{1}, T_{2}\right)$ defined in $(5.5)$ is

$$
F\left(t, T_{1}, T_{2}\right)=\exp \left(-\frac{1}{2} \int_{0}^{t} \bar{\Sigma}^{2}\left(s, T_{1}, T_{2}\right) d s+\int_{0}^{t} \bar{\Sigma}\left(s, T_{1}, T_{2}\right) d W(s)\right) .
$$

For the no-arbitrage condition to hold, we need that the swap model allows for a forward dynamics $F(t, u, u)$. A necessary condition for this is that

$$
\lim _{T_{2} \downarrow T_{1}} \bar{\Sigma}\left(t, T_{1}, T_{2}\right)=\bar{\Sigma}\left(t, T_{1}, T_{1}\right),
$$

which we now assume to hold. Then, after multiplying by $T_{2}-T_{1}$ on both sides of (5.2) followed by differentiating with respect to $T_{2}$, we find the relation

$$
\begin{gathered}
F\left(t, T_{1}, T_{2}\right)\left\{\frac{1}{T_{2}-T_{1}}-\int_{0}^{t} \bar{\Sigma}\left(s, T_{1}, T_{2}\right) \frac{\partial}{\partial T_{2}} \bar{\Sigma}\left(s, T_{1}, T_{2}\right) d s+\int_{0}^{t} \frac{\partial}{\partial T_{2}} \bar{\Sigma}\left(s, T_{1}, T_{2}\right) d W(s)\right\} \\
=\frac{1}{T_{2}-T_{1}} F\left(t, T_{2}, T_{2}\right) .
\end{gathered}
$$

We have appealed to the stochastic integration-by-parts formula for the Wiener integral in order to exchange the order of differentiation and stochastic integration. Now, observe that the right hand side of (5.7) is positive. The left hand side, however, has positive probability of becoming negative, since

$$
\int_{0}^{t} \frac{\partial}{\partial T_{2}} \bar{\Sigma}\left(s, T_{1}, T_{2}\right) d W(s)
$$


can attain all values on the real line. The only exception is when

$$
\frac{\partial}{\partial T_{2}} \bar{\Sigma}\left(t, T_{1}, T_{2}\right)=0
$$

that is, when the volatility does not depend on the terminal time of maturity. Hence, if the volatility depends on $T_{2}$, the no-arbitrage condition (5.2) can not hold. An analogous argument gives the same conclusion if the volatility depends on $T_{1}$. We therefore see that the lognormal model can not satisfy the no-arbitrage condition and at the same time possess a volatility structure depending on the delivery period. In conclusion, the only lognormal model which yields an arbitrage-free dynamics is the Black-76 model.

The model suggested by Black [8] in 1976 is still very popular among practitioners in various energy and commodity markets, and the electricity market is no exception. One feature of the volatility documented in variuous commodity and electricity markets is the maturity effect. Volatility tends to increase as time to maturity decreases. Samuelson [49] claimed that the volatility of futures price returns increases as time to maturity decreases. He argued that the most important information was revealed close to maturity of the contract. For example, the weather affecting demand or a temporary supply disruption will affect spot prices and hence short dated futures contracts. Short-term price movements are not expected to persist, but rather revert back towards a normal level. This implies that long dated contracts will be less affected by spot price changes and experience lower volatility than short dated contracts.

Some researchers analyzing agricultural commodities have focused on seasonality, which is a calendar effect. For crop commodities one would typically expect the information flow to vary during the crop cycle. The most important information is revealed during the growth and harvest season, hence seasonality in the volatility of futures prices is expected. Bessembinder et al. [6] have shown formally that the maturity effect implies mean reversion in the underlying spot price. Galloway and Kolb [21] concluded that the maturity effect is present in markets where commodities experience seasonal demand and/or supply, but not in commodity markets where the cost-of-carry model works well. In electricity markets the cost of carry model is of little use since electricity cannot be stored. In most electricity markets demand is seasonal, and especially in hydro power based system, so is supply. This means that both seasonality and maturity effects are potentially important in electricity markets. The seasonality effect can be modelled by for instance choosing $\bar{\sigma}(t)$ to be a truncated Fourier series of sines and cosines.

The maturity effect is extremely pronounced in the electricity market. This means that a realistic specification of the swap dynamics should include a maturity dependent volatility. As we have seen, the lognormal dynamics (5.5) does not permit such a volatility depending on the delivery time, $\bar{\Sigma}\left(t, T_{1}, T_{2}\right)$. However, this restriction is a consequence of the assumption that we want to model the swap dynamics consistently in an arbitrage-free 
way for all theoretically possible delivery periods. In practice, the market trades in a finite number of different swaps, and the modeling approach taken in this Section will be rather extreme compared to the actual financial products we are interested in modeling. In the next Section we shall relax the no-arbitrage condition (5.2) to hold only for traded swaps, and in this way open up for lognormal models with a desirable volatility structure.

\section{SWAP MOdELING USING THE HJM-APPROACH: A MARKET MODEL}

The strong requirement on $F\left(t, T_{1}, T_{2}\right)$ in (5.2) makes it attractive to first model the forward dynamics, and then integrate up to achieve the swap dynamics (as we described in Section 4 above). However, as we have already discussed, this approach involves smoothing of data where a lot of information is changed in a rather complicated way. In addition, the resulting swap dynamics becomes very complicated, even for natural lognormal or multiplicative specifications of the forward, and is not easily tractable for risk management or option pricing analysis. In this section we discuss an alternative version of the HJMapproach where we can use the market price observations directly to fit models and at the same time avoid arbitrage possibilities in the specification.

From a market point of view, the condition (5.2) is far too restrictive since the number of swap contracts that overlap in reality is rather small. In fact, considering our data sample of 55712 prices from Nord Pool (see Section 8), only 1793 or about $3.2 \%$ overlap. A simple way of attacking the modeling of swaps is to restrict the attention to the "basic building blocks" of the market. By this we mean to single out all the swaps that can not be decomposed into other traded swap contracts, and model these by a stochastic dynamical model. These swaps being the "basic building blocks" will be referred to as the atomic swaps of the market. Given the dynamics of the atomic swaps, we use the discrete version of the no-arbitrage condition (5.1) to derive the dynamics of the swaps that can be composed by different atomic swaps. If for instance the market trades in monthly and quarterly swaps, the monthly swaps will become the atoms. Letting $T_{1}, T_{2}, \ldots, T_{12}$ denote the first day of each month, we can model the atomic swaps by the pricing dynamics

$$
d F\left(t, T_{i}, T_{i+1}\right)=\Sigma\left(t, T_{i}, T_{i+1}\right) d W(t)+\int_{\mathbb{R}} \Psi\left(t, T_{i}, T_{i+1}, z\right) \tilde{N}(d z, d t)
$$

for $i=1, \ldots, 11$ and $t \leq T_{i}$. The quarterly swap dynamics will then become, for the first quarter say,

$$
F\left(t, T_{1}, T_{4}\right)=\sum_{i=1}^{3} w_{i} F\left(t, T_{i}, T_{i+1}\right),
$$

where we recall that $w_{i}=\int_{T_{i}}^{T_{i+1}} w(u) d u / \int_{T_{1}}^{T_{4}} w(u) d u$.

Choosing to model in this way enables us to state simple dynamical models for the swap price which satisfy the no-arbitrage condition (5.1). In practice, we can only use the price data for the atomic swaps in the market when fitting the model, and have to throw away 
the data for all other contracts. However, not much information is lost since the market already has taken into account the arbitrage opportunities.

An unfortunate side effect of our modeling approach is that we loose the mathematical connection to spot prices completely. Since in general there exist no natural forward dynamics connected to this modeling approach (if one would exist, we are led to a model where the condition (5.2) holds) we can not talk about any spot dynamics either. However, the spot market can be interpreted as a market for swap contracts with delivery in a specified hour of the next day. In this sense, we can use our (fitted) swap model to impose a (risk-neutral) dynamics for the spot. Moreover, for doing risk management on portfolios of swaps, or pricing options written on swaps, our version of the HJM modeling approach is sufficient. Below we will discuss the volatility structure for lognormal swap models and the specification of a multiplicative dynamics with jumps.

6.1. Lognormal models. We consider in this Subsection some lognormal specifications of the swap dynamics. By ignoring the Lévy term in the pricing dynamics, we will not be able to model abrupt shifts or jumps in the market. The possibility to model leptokurtic returns are also limited as long as we do not open for a Lévy process. In a lognormal model, the volatility is the key parameter. We shall consider different one-factor models inspired from forward curve models. Note also that our lognormal models of the swap rate dynamics are intended for the swap atoms, and not all the swaps traded.

Throughout this Section, we choose $w(u)=1$, which means settlement at maturity and not continuously. Hence, $\widehat{w}\left(u ; T_{1}, T_{2}\right)=1 /\left(T_{2}-T_{1}\right)$. The case $w(u)=\exp (-r u)$ is easily implemented by straightforward modifications of the theory to be presented. From discussions in the previous section, we argued that the maturity effect is pronounced in the electricity market and realistic lognormal models must include a volatility which depends on the period of delivery. To create lognormal models for the swap dynamics which have the potential to catch up this maturity effect, we use inspiration from the volatility specifications in forward models already considered in the literature for commodity markets.

Suppose that the swap dynamics is given by

$$
d F\left(t, T_{1}, T_{2}\right)=\bar{\Sigma}\left(t, T_{1}, T_{2}\right) F\left(t, T_{1}, T_{2}\right) d W(t),
$$

where $\bar{\Sigma}\left(t, T_{1}, T_{2}\right)$ is a continuously differentiable, and positive function. For a given forward volatility, $\bar{\sigma}(t, u)$, we can associate a swap volatility as:

$$
\bar{\Sigma}\left(t, T_{1}, T_{2}\right)=\frac{1}{T_{2}-T_{1}} \int_{T_{1}}^{T_{2}} \bar{\sigma}(t, u) d u .
$$

The reader should note that the sole purpose of (6.2) is to create natural swap volatility structures, and not to try to derive the swap dynamics from the forward.

In the next Subsections we propose different swap curve volatility models and discuss their properties and relation to forward models. 
6.1.1. Swap volatility models with exponential decay. Schwartz [50] suggests several models for the spot rate of oil. His simple one-factor model implies a futures price dynamics with volatility given as

$$
\bar{\sigma}(t, u)=a e^{-b(u-t)},
$$

where $a, b \geq 0$ are constants. Derived from a spot model, Lucia and Schwartz [42] obtain this volatility structure for their forward curve dynamics. We note that the maturity effect is modeled as a negative exponential function. This is common for all the models we review in this subsection. The basic constant volatility model suggested by Black [8] appears if $b=0$. From (6.2) we propose a one factor "Schwartz" swap model

$$
\bar{\Sigma}\left(t, T_{1}, T_{2}\right)=a \phi\left(T_{1}, T_{2}\right),
$$

where

$$
\phi\left(T_{1}, T_{2}\right)=\frac{e^{-b\left(T_{1}-t\right)}-e^{-b\left(T_{2}-t\right)}}{b\left(T_{2}-T_{1}\right)} .
$$

Schwartz' model picks up the maturity effect, but not the seasonality. Fackler and Tian [19] modify Schwartz' model to also include seasonality, and they show that seasonality is an important feature of volatility in the futures market for soy beans. Their spot price model implies futures price volatility of the type

$$
\bar{\sigma}(t, u)=a(t) e^{-b(u-t)},
$$

where the term $a(t)$ picks up seasonality. The seasonal part can be modeled as a truncated Fourier series

$$
a(t)=a+\sum_{j=1}^{J}\left(d_{j} \sin (2 \pi j t)-f_{j} \cos (2 \pi j t)\right),
$$

where $t$ is measured in years ${ }^{6}$. In this model the parameters are restricted in the following way: $d_{j}$ and $f_{j}$ are real constants and $a, b \geq 0$. We see that as $u \rightarrow t$, the volatility function collapses to $\bar{\sigma}(t, t)=a(t)$. This means that $a(t)$ governs the implied spot price volatility. Our suggested swap volatility model is

$$
\bar{\Sigma}\left(t, T_{1}, T_{2}\right)=a(t) \phi\left(T_{1}, T_{2}\right) .
$$

We note that the maturity effect is modeled the same way in Fackler and Tian [19] and Schwartz [50].

In electricity markets, volatility raises sharply as contracts approaches maturity. This can be achieved by a high value of $b$ in the models above. But this again implies that the prices of futures contracts with long time to maturity gets very low, and a pure negative exponential maturity effect may have a hard time capturing the volatility of both short

\footnotetext{
${ }^{6}$ If time is measured on a different scale, we must change the frequency appropriately
} 
and long term contracts. For this reason Strickland [55] proposed the following volatility function in a forward curve model:

$$
\bar{\sigma}(t, u)=a\left((1-c) e^{-b(u-t)}+c\right) .
$$

The parameters are restricted in the following way: $a, b \geq 0$ and $0 \leq c \leq 1$. In the limit, as $u$ approaches infinity, we note that $\bar{\sigma}(t, u) \rightarrow a c$, and as $u$ approaches $t, \bar{\sigma}(t, u) \rightarrow a$. Thus, in this specification the volatility of a futures contract is bounded within $[a c, a]$. If for example $c=0.5$, short term volatility doubles the long run volatility. The associated swap volatility model becomes

$$
\bar{\Sigma}\left(t, T_{1}, T_{2}\right)=a\left((1-c) \phi\left(T_{1}, T_{2}\right)+c\right),
$$

where $\phi$ is defined in (6.5) .

Koekebakker and Lien [37] combine the model suggested by Strickland [55] with seasonal spot volatility. They propose the the following forward curve volatility function:

$$
\bar{\sigma}(t, u)=a(t)\left((1-c) \mathrm{e}^{-b(u-t)}+c\right)
$$

with parameter restrictions $a, b \geq 0,0 \leq c \leq 1, d_{j}$ and $f_{j}$ being constants. The swap volatility model becomes in this case

$$
\bar{\Sigma}\left(t, T_{1}, T_{2}\right)=a(t)\left((1-c) \phi\left(T_{1}, T_{2}\right)+c\right) .
$$

We suggest the following model that has not been investigated previously:

$$
\bar{\Sigma}\left(t, T_{1}, T_{2}\right)=a \phi\left(T_{1}, T_{2}\right)+c(t),
$$

The corresponding forward curve model is

$$
\bar{\sigma}(t, u)=a e^{-b(u-t)}+c(t)
$$

with $c(t)$ given as in (6.7). We note that this model has a clear separation of the seasonaland maturity effects. The implied spot volatility is $a+c(t)$ and long run volatility is governed by $c(t)$ alone. As it turns out, this separation of maturity- and seasonality is very much in agreement with data.

The six different swap volatility models and the forward curve model that "inspired" them are summarized in Table 2, where we have named them E1 to E6, respectively for later reference. We will provide parameter estimates for all these models in Section 8

6.2. A multiplicative model with jumps. We include a discussion of a multiplicative jump model for the swap rate dynamics where the whole swap dynamics is allowed to make a sudden shift at random times. The model is a direct generalization of Merton's jump diffusion dynamics for stock prices introduced in [43], and later applied to energy spot modeling in Clewlow and Strickland [13]. 


\begin{tabular}{cll} 
Model & \multicolumn{1}{c}{$\bar{\sigma}(t, u)$} & \multicolumn{1}{c}{$\left(t, T_{1}, T_{2}\right)$} \\
\hline E1 & $a$ & $a$ \\
E2 & $a e^{-b(u-t)}$ & $a \phi\left(T_{1}, T_{2}\right)$ \\
E3 & $a(t) e^{-b(u-t)}$ & $a(t) \phi\left(T_{1}, T_{2}\right)$ \\
E4 & $a\left((1-c) e^{-b(u-t)}+c\right)$ & $a\left((1-c) \phi\left(T_{1}, T_{2}\right)+c\right)$ \\
E5 & $a(t)\left((1-c) e^{-b(u-t)}+c\right)$ & $a(t)\left((1-c) \phi\left(T_{1}, T_{2}\right)+c\right)$ \\
E6 & $a e^{-b(u-t)}+c(t)$ & $a \phi\left(T_{1}, T_{2}\right)+c(t)$
\end{tabular}

TABLE 2. Swap volatility models with exponential decaying volatility. The volatility dynamics of the forward models are used as a basis, reported in the first column in the table under the coding E1=Black (1976), E2=Schwartz (1997), E3=Strickland (2002), E4=Fackler and Tian (1999), E5=Koekebakker and Lien (2004) and E6=Our suggested model. The second column represents our swap curve volatility obtained from (6.2). The parameters $a, b$ and $c$ are constants, while specification of $a(t)$ (and $c(t))$ and $\phi\left(T_{1}, T_{2}\right)$ are given in (6.7) and (6.5), respectively.

Let $X_{1}, X_{2}, \ldots$ be a sequence of independent, identically distributed random variables, each with a probability distribution $\nu(d z)$ on $\mathbb{R}$. Assume that $\mathbb{E}\left[\left|X_{i}\right|\right]<\infty$. Introduce a Poisson process $N(t)$ with jump intensity $\lambda$, and define the compound Poisson process

$$
L(t)=\sum_{i=1}^{N(t)} X_{i},
$$

with Lévy measure given by $\ell(d z)=\lambda \nu(d z)$. Note that the compensator of of $L(t)$ is equal to $\lambda \int_{\mathbb{R}} z \nu(d z) d t=\lambda \mathbb{E}\left[X_{i}\right] d t$.

With all these definitions at hand, we consider the following multiplicative model for the swap prices

$$
\begin{aligned}
d F\left(t, T_{1}, T_{2}\right)= & F\left(t-, T_{1}, T_{2}\right)\left\{-\lambda \kappa \bar{\Psi}\left(t, T_{1}, T_{2}\right) d t+\bar{\Sigma}\left(t, T_{1}, T_{2}\right) d W(t)\right. \\
& \left.+\bar{\Psi}\left(t, T_{1}, T_{2}\right) X d N(t)\right\} .
\end{aligned}
$$

Here, $X$ is distributed as $\nu(d z)$, and $\bar{\Sigma}, \bar{\Psi}$ are the volatility functions for the Wiener and jump part, respectively. We have defined $\kappa:=\mathbb{E}[X]$. Both volatility functions are deterministic, being continuous and bounded with respect to all arguments. In order to assure that the swap process stays positive throughout its lifetime, we suppose that the distribution of the $X_{i}$ 's is such that $\bar{\Psi}\left(t, T_{1}, T_{2}\right) X_{i}>-1$. This condition ensures positivity of the prosess defined in (6.13) (see Protter [47]). 
The multiplicative model (6.13) is a direct extension of the lognormal model. Note that we have used the compensated version of $L(t)$ in the modeling of the jump part, so that the martingale property of the swap dynamics is preserved.

The rationale behind the multiplicative model is as follows: in times of normal market behaviour, the swap dynamics evolves according to a geometric Brownian motion. Abrupt changes in the market are modeled by jumps arriving at exponentially distributed random times, and the jumps are of random size given by $X$. Sudden information affects the swaps with short time to maturity and short delivery period the most. Swaps with longer time to delivery are less affected, and this is reflected in a "volatility" function $\bar{\Psi}$, which can be close to one for $T_{1}$ close to current time, while close to zero when the delivery period starts farer away. In this paper we shall not analyse this model empirically, but leave the statistical issues for later studies.

\section{PRICING AND HEDGING OF OPtIONS WRITTEN ON SWAPS}

In order to derive the fair price and hedging strategy for an option on a swap, we need to have an underlying dynamics of the swap price dynamics. Consider a call option with strike $K$ and exercise time $T$ written on a swap with delivery over $\left[T_{1}, T_{2}\right]$, where $T \leq T_{1}$. The arbitrage free price at time $t \leq T$ of this option will be

$$
P(t)=\mathrm{e}^{-r(T-t)} \mathbb{E}\left[\left(F\left(T, T_{1}, T_{2}\right)-K\right)^{+} \mid \mathcal{F}_{t}\right] .
$$

The expectation is taken under a risk neutral probability $Q$, which is the measure under which we have stated all our models. If we know the dynamics of $F$, we can now start to derive the option price $P(t)$. Furthermore, hedging measures like for instance delta, gamma or vega can in principle be found by differentiating with respect to the initial swap curve or volatility.

We have presented two different approaches to model the swap dynamics in the HJMframework, where the former starts out with a stochastic modeling of fixed-delivery forwards, while the latter models the swap prices directly. For the direct model, we have discussed a "market approach", which concentrates on modeling only those swaps actually traded. In the framework of modeling the fixed-delivery forwards, it becomes a cumbersome task to calculate both option prices and risk measures for hedging, whereas in the framework of a direct HJM-approach to swap price modeling the option pricing problem is simpler to handle. In fact, we obtain a Black-76 type of formula with the latter.

We now analyze the problem of pricing options within the two modeling frameworks.

7.1. HJM-approach for fixed-delivery forward dynamics. Assume we have a model for the fixed-delivery forward $f(t, u)$ given as in (3.2). The price of the call option in (7.1) 
becomes

$$
C(t)=\mathrm{e}^{-r(T-t)} \mathbb{E}\left[\left(\int_{T_{1}}^{T_{2}} \widehat{w}\left(u ; T_{1}, T_{2}\right) f(T, u) d u-K\right)^{+} \mid \mathcal{F}_{t}\right]
$$

As we have seen, the dynamics for the swap contract becomes involved, even when we specialize the dynamics for $f$ to be lognormal. Thus, we need to approach the pricing problem by numerical procedures or invoke an approximation of the swap price at maturity as proposed by Bjerksund et al. [9] in the lognormal case.

Instead of approximating the dynamics, we will here discuss and outline how the option price can be derived by using a Monte Carlo method when the forward price $f(t, u)$ follows a lognormal dynamics

$$
d f(t, u)=\bar{\sigma}(t, u) f(t, u) d W(t)
$$

with $\bar{\sigma}$ being a bounded and continuous deterministic function. To find $C(t)$, we need to simulate the random variable

$$
\int_{T_{1}}^{T_{2}} \widehat{w}\left(u ; T_{1}, T_{2}\right) f(T, u) d u
$$

and then find the conditional expectation with respect to the $\sigma$-algebra $\mathcal{F}_{t}$. To simplify matters, let us focus on the price at time 0 , that is, at $P(0)$.

We face the problem of choosing an appropriate numerical integration routine in order to approximate the random variable in (7.3). However, the definition of a swap contract guides us in this matter, since in practice a swap is settled against the hourly day-ahead prices, hence the integral is in fact a mathematical approximation of the real world contract looking like

$$
\sum_{i=1}^{n} \widehat{w}\left(u_{i} ; T_{1}, T_{2}\right) f\left(T, u_{i}\right) \approx \int_{T_{1}}^{T_{2}} \widehat{w}\left(u ; T_{1}, T_{2}\right) f(T, u) d u
$$

where $T_{1}<u_{1}<\ldots<u_{n-1}<u_{n}=T_{2}$ are the hours in the interval $\left[T_{1}, T_{2}\right]$ and $u_{i}-u_{i-1}=1$ hour. Thus, we use a simple Riemann approximation for this integral. To this end, observe that

$$
\begin{aligned}
f\left(T, u_{i}\right) & =f\left(0, u_{i}\right) \exp \left(-\frac{1}{2} \int_{0}^{T} \bar{\sigma}^{2}\left(t, u_{i}\right) d t+\int_{0}^{T} \bar{\sigma}\left(t, u_{i}\right) d W(t)\right) \\
& =f\left(0, u_{i}\right) \exp \left(-\frac{1}{2} \int_{0}^{T} \bar{\sigma}^{2}\left(t, u_{i}\right) d t+\sqrt{\int_{0}^{T} \bar{\sigma}^{2}\left(t, u_{i}\right) d t} \cdot X\right)
\end{aligned}
$$


where the second equality holds in distributional sense with $X$ being a standard normally distributed random variable. Hence, we can rewrite the price $C(0)$ as follows: ${ }^{7}$

$$
C(0) \approx \mathrm{e}^{-r T} \mathbb{E}\left[(H(X)-K)^{+}\right]
$$

where

$$
H(X)=\sum_{i=1}^{n} \widehat{w}\left(u_{i} ; T_{1}, T_{2}\right) f\left(0, u_{i}\right) \exp \left(-\frac{1}{2} \int_{0}^{T} \bar{\sigma}^{2}\left(t, u_{i}\right) d t+\sqrt{\int_{0}^{T} \bar{\sigma}^{2}\left(t, u_{i}\right) d t} \cdot X\right)
$$

The Monte Carlo algorithm will now simply be to

(1) Draw $N$ samples from $X: x_{1}, \ldots, x_{N}$

(2) Calculate

$$
C(0) \approx \mathrm{e}^{-r T} \frac{1}{N} \sum_{k=1}^{N}\left(H\left(x_{k}\right)-K\right)^{+}
$$

Let us move our attention to the question of hedging this call option. We know from the general arbitrage theory that the call option in our situation can be perfectly hedged using the underlying swap contract. The standard way to derive the hedge ratio, also known as the option's delta, is to differentiate the option price with respect to the underlying asset. In this case, this entails in finding $\partial C(0) / \partial F\left(0, T_{1}, T_{2}\right)$. Unfortunately, this is a non-trivial task, since the dependency on $F\left(0, T_{1}, T_{2}\right)$ is not at all explicit, but only implicitly given through the relation

$$
F\left(0, T_{1}, T_{2}\right)=\int_{T_{1}}^{T_{2}} \widehat{w}\left(u, T_{1}, T_{2}\right) f(0, u) d u .
$$

What is possible, is to find $\partial C(0) / \partial f(0, u)$ for a given $u \in\left[T_{1}, T_{2}\right]$, and this may give the starting point for deriving an approximative hedge by for instance averaging over these derivatives. By the Riemann approximation, we see that $C(0)$ is indeed a function of $f\left(0, u_{i}\right), i=1, \ldots, n$. Hence, from the chain rule we have

$$
\frac{\partial C(0)}{\partial F\left(0, T_{1}, T_{2}\right)} \approx \sum_{i=1}^{n} \frac{\partial C(0)}{\partial f\left(0, u_{i}\right)} \frac{\partial f\left(0, u_{i}\right)}{\partial F\left(0, T_{1}, T_{2}\right)}
$$

We know that

$$
\frac{\partial F\left(0, T_{1}, T_{2}\right)}{\partial f\left(0, u_{i}\right)}=\widehat{w}\left(u_{i} ; T_{1}, T_{2}\right)
$$

and treating these differentials as incremental changes, we see that

$$
\frac{\partial C(0)}{\partial F\left(0, T_{1}, T_{2}\right)} \approx \sum_{i=1}^{n} \frac{\partial C(0)}{\partial f\left(0, u_{i}\right)} \frac{1}{\widehat{w}\left(u_{i} ; T_{1}, T_{2}\right)} .
$$

\footnotetext{
${ }^{7}$ Note that this price is approximative if we insist on defining $C(0)$ via the integral connection between the forward and the swap, whereas it is indeed the price if we consider the actual swap contracts in the market starting out with a fixed-delivery forward model.
} 
However, the difficulty in explicitly finding a hedge may be another argument against modeling the fixed-delivery forwards instead of the swaps directly, the case we now consider.

7.2. HJM-approach for swap dynamics. In this section we consider pricing of call options on swaps when we have a stochastic dynamics of the swap given by (3.1). It turns out that the option price can be conveniently expressed by using the change of numéraire technique developed by Geman et al. [22], where a so-called forward measure is introduced. In our context this is naturally denoted a swap measure, defined as

$$
d Q^{\mathrm{SW}}=L(T) d Q \text { on } \mathcal{F}_{T}
$$

where the density process of the Radon-Nikodym derivative $L(T)$ is

$$
L(t)=\frac{F\left(t, T_{1}, T_{2}\right)}{F\left(0, T_{1}, T_{2}\right)} .
$$

Under the conditions on the dynamics (3.1), we know that $F\left(t, T_{1}, T_{2}\right)$ is a square integrable martingale, and hence, $L(t)$ is a martingale. Thus, the probability measure $Q^{\mathrm{SW}}$ is a martingale measure. We have the following general pricing equation for a European call option (see Appendix B for a proof):

Theorem 7.1. The price $C(t)$ at time $t$ of a European call option with maturity $T \leq T_{1}$ and exercise price $K$ written on a swap contract with delivery period $\left[T_{1}, T_{2}\right], T_{1}<T_{2}$ is given by the formula

$$
C(t)=e^{-r(T-t)}\left\{F\left(t, T_{1}, T_{2}\right) Q^{S W}\left(F\left(T, T_{1}, T_{2}\right) \geq K \mid \mathcal{F}_{t}\right)-K Q\left(F\left(T, T_{1}, T_{2}\right) \geq K \mid \mathcal{F}_{t}\right)\right\} .
$$

In the case of a lognormal specification of $F$, i.e.,

$$
d F\left(t, T_{1}, T_{2}\right)=\bar{\Sigma}\left(t, T_{1}, T_{2}\right) F\left(t, T_{t}, T_{2}\right) d W(t),
$$

we can derive an explicit Black-76 type of pricing formula. The swap measure will in this case have a Radon-Nikodym derivative given by

$$
L_{t}^{F}=\exp \left(-\frac{1}{2} \int_{0}^{t} \bar{\Sigma}^{2}\left(s, T_{1}, T_{2}\right) d s+\int_{0}^{t} \bar{\Sigma}\left(s, T_{1}, T_{2}\right) d W(s)\right)
$$

A straightforward calculation yields the formula

Corollary 7.2. When $F$ follows a lognormal dynamics with volatility $\bar{\Sigma}\left(t, T_{1}, T_{2}\right)$ being bounded and continuous, the price of a call option with maturity $T \leq T_{1}$ and exercise price $K$ will be

$$
C(t)=e^{-r(T-t)}\left\{F\left(T, T_{1}, T_{2}\right) N\left(d_{1}\right)-K N\left(d_{2}\right)\right\}
$$


where $N(d)$ is the cumulative probability distribution function for a standard normal variable and

$$
\begin{aligned}
& d_{1}=\frac{\ln \left(F\left(t, T_{1}, T_{2}\right) / K\right)+\frac{1}{2} \int_{t}^{T} \bar{\Sigma}^{2}\left(s, T_{1}, T_{2}\right) d s}{\sqrt{\int_{t}^{T} \bar{\Sigma}^{2}\left(s, T_{1}, T_{2}\right) d s}} \\
& d_{2}=d_{1}-\sqrt{\int_{t}^{T} \bar{\Sigma}^{2}\left(s, T_{1}, T_{2}\right) d s}
\end{aligned}
$$

Also to find the hedge is in this case quite simple when we have a lognormal swap dynamics.

If there are overlapping swaps that need to be modeled as a sum of atomic swaps, we will no longer have the explicit Black-76 formula in the Corollary above for an option written on this, since a sum of lognormal variables is not lognormal. Then again we need to apply numerical procedures in order to find a price.

Finally, let us remark the resemblance with the suggestion of Bjerksund et al. [9], who proposes a lognormal approximation of the swap dynamics from a lognormal model of the fixed-delivery forward dynamics. They, too, will have a model which can be used to price options with a Black-76 type of formula as above. Hover, the essential difference with our approach is that we base our volatility model on data directly, and not on a mathematical approximation from a model which is fitted using smoothed data.

\section{EMPIRICAL ANALYSIS OF ELECTRICITY CONTRACTS TRADED ON NORDPOOL}

In this Section we describe the data collected from Nord Pool, our estimation method and the results from fitting to observed data.

8.1. Data description and descriptive statistics. From Nord Pool we obtained closing prices for all contracts traded from September 25, 1995 untill March 26, 2004. Information regarding settlement periods were also obtained from Nord Pool's database. The power contracts refer to $1 \mathrm{MW}$ load during every hour (base load) for a given delivery period. The contracts are settled against the realised day-ahead prices in the delivery period. The trading period stops when the contracts enter the delivery period. The size and trading period varies considerably for the contracts available. We will give a brief description of daily, weekly, monthly, seasonal and yearly contracts below.

The daily contracts were introduced in September 1999. Our first observation is from September 3, 1999. The daily contracts have delivery period each day of the week. On Monday there exist six daily contracts; Tuesday, Wednesday, Thursday, Friday, Saturday and Sunday. The 24 hour delivery period of the Tuesday contract starts midnight Monday, and the contract expires at the end of the trading day on Monday. Each day a new contract expires. On Thursday only contracts for Friday, Saturday and Sunday exist. Seven new 
daily contracts are introduced Friday morning, the Monday contract being the one with with shortest time to delivery. All these new contracts are traded on Friday, along with the nearby Saturday and Sunday contracts. A total of 9 contracts. Note that Friday is the only day that the Monday contract is traded. This means that our data set does not allow us to compute daily return on Monday contracts.

The weekly contracts are specified with a delivery period of 7 days (168 hours). The delivery period starts Sunday at midnight and ending midnight the following Sunday. The contract with delivery the following week expires the preceding Friday. A maximum of 7 contracts and a minimum of 4 contracts are traded each week. New contracts are introduced every fourth Monday.

Block contracts are futures-type swaps. In 2003 block contracts have been replaced with monthly contracts, with delivery periods in each of the twelve months in a year. Also, all new contracts introduced in 2003 and later are denominated in EUR. These contracts were not traded in the month prior to delivery. Each month one contract expires and a new is introduced. Since these contracts have delivery period of 28 days (4 weeks), each year is divided into 13 block contracts, 10 of which is traded simultaneously. Since $4 \times 28 \times 13=364$, this does not add up to one year. The last block contract of 1998 had a delivery period of 35 days to make sure that the delivery period of the first block contract in January 1999 had delivery period starting January 1. To avoid these problems, and to offer products more similar to contracts trading at other commodity exchanges, the block contracts was replaced in 2003 by contracts with delivery periods equal to calendar months. Also, all new contracts introduced in 2003 and later on are denominated in EUR.

The seasonal contracts are forward-style swaps, and so are the yearly contracts described below. The contract specifications of the seasonal contracts have gone from a 3 year seasonal structure to a quarterly structure. Previously each year was divided into three seasons: V1 - late winter (January 1 - April 30), S0 - summer (May 1 - September 30) and V2 - early winter (October 1 - December 31). From 1995 to the end of 1999 the seasonal contract were marked-to-market futures-style swap contracts. In 1997, seasonal forwardstyle swap contracts were introduced. Both contract types were listed in the 1997-1999 period. From 2000 until the end of the sample the seasonal contracts are all forward-style. The first quarterly seasonal contracts were listed January 2, 2004 for each quarter of the year 2006. These contracts are denominated in EUR, and all new seasonal contracts will be of this type, eventually replacing the old seasonal contracts.

Yearly contracts have delivery period of one year. These contracts started trading in 1998. Yearly contracts for the following 3 years are available for trading. These contracts have delivery period of $24 \times 365=8760$ hours (8784 hours in leap years). Each new contract is traded for 3 years, until it expires in late december prior to the start of the delivery period January 1. Each January a new yearly contract is introduced with delivery period starting in 3 years. 
Mean Nobs. Std. Min. Max. $\overline{D P} \overline{T T D}$

Panel A: Full sample

$\begin{array}{lllllll}178.0 & 55712 & 74.00 & 30.0 & 1425.0 & 72 & 230\end{array}$

Panel B: Sub-samples according to DP

\begin{tabular}{lrrrrrrr}
\hline Day & 195.3 & 6214 & 110.68 & 30.0 & 1425.0 & 1 & 4 \\
Week & 184.3 & 11620 & 97.56 & 46.3 & 885.0 & 7 & 22 \\
Month & 174.9 & 17818 & 68.40 & 70.0 & 750.0 & 28 & 155 \\
Season & 172.2 & 15887 & 41.28 & 81.5 & 634.0 & 120 & 474 \\
Year & 169.3 & 4173 & 33.28 & 120.6 & 380.0 & 365 & 538
\end{tabular}

TABLE 3. Descriptive statistics of swap price levels. The full sample consists of daily closing prices on financial elctricity contracts listed on Nord Pool in the period September 25, 1995 untill March 26, 2004. The contracts are quoted in NOK/MWh, and each day in the delivery period represent 24 MWh. The columns $\overline{D P}$ and $\overline{T T D}$ are average delivery period and average time to delivery, respectively.

8.1.1. Descriptive statistics of price levels. The total sample consists of 55712 observation. Contracts denominated in EUR are transformed to NOK using the exchange rate the relevant date. Exchange rates are collected from Norges Bank ${ }^{8}$. For each contract we observe the information provided in Table 1; time of year, closing price (measured in NOK/MWh), time to delivery (TTD) and start and end of the delivery period (DP). We need to know the time of year the observation is made in order to include seasonality in our models. Descriptive statistics for the contract price levels are given in Table 3, Panel A. Mean price is 178, minimum price is 30 and maximum price is 1425 . This suggest highly right-skewed distribution of price levels. In Panel B we sort the data in different sub-samples accoording the size of the delivery period. Note that $\overline{D P}$ and $\overline{T T D}$ indicate the average delivery period and time to delivery respectively. We note that the right-skewed price distribution is more pronounced the shorter the time to maturity (and consequently the longer delivery period). This observation confirms the finding in Longstaff and Wang [41] that the right-skewedness is more pronounced in the real-time market than in the (more distant) day-ahead market. In Panel B we also note that along with rightskewedness the standard deviations and average price levels are decreasing in TTD and

\footnotetext{
${ }^{8}$ Norges Bank is the central bank of Norway: www.norgesbank.no
} 
Mean Nobs. Std. Min. Max. $\overline{D P} \overline{T T D}$

Panel A: Full sample

\begin{tabular}{llllllll}
\hline All & -0.001 & 49425 & 0.502 & -0.66 & 0.69 & 72 & 230
\end{tabular}

Panel B: Sub-samples sorted according to DP

\begin{tabular}{llllllrr}
\hline Day & -0.005 & 4543 & 1.116 & -0.66 & 0.66 & 1 & 4 \\
Week & -0.002 & 11179 & 0.547 & -0.39 & 0.41 & 7 & 22 \\
Month & 0.000 & 17703 & 0.389 & -0.31 & 0.69 & 28 & 155 \\
Season & 0.000 & 14052 & 0.217 & -0.28 & 0.34 & 120 & 474 \\
Year & 0.000 & 1948 & 0.182 & -0.12 & 0.16 & 365 & 538
\end{tabular}

Panel C: Sub-samples sorted according to TTD

\begin{tabular}{lrrrrrrr}
\hline$[0,2$ mon. $\rangle$ & -0.002 & 18291 & 0.724 & -0.66 & 0.69 & 16 & 19 \\
{$[2$ mon., 6 mon. $)$} & 0.000 & 9525 & 0.404 & -0.31 & 0.69 & 58 & 120 \\
{$[6$ mon., 1 year $\rangle$} & 0.000 & 9969 & 0.296 & -0.28 & 0.34 & 80 & 256 \\
{$[1$ year, $->\rangle$} & 0.000 & 11428 & 0.188 & -0.28 & 0.28 & 171 & 650
\end{tabular}

Panel D: Sub-samples sorted according to time of year

$\begin{array}{lrllllll}\text { 1. quarter } & -0.002 & 12847 & 0.571 & -0.66 & 0.69 & 74 & 265 \\ \text { 2. quarter } & 0.000 & 11400 & 0.463 & -0.51 & 0.28 & 71 & 242 \\ \text { 3. quarter } & 0.000 & 11682 & 0.464 & -0.44 & 0.60 & 76 & 211 \\ \text { 4. quarter } & 0.000 & 13496 & 0.493 & -0.40 & 0.41 & 68 & 204\end{array}$

TABle 4. Descriptive statistics of swap price returns. The full sample consists of daily closing prices on financial elctricity contracts listed on Nord Pool in the period 25.09.1995 - 26.03.2004. The columns $\overline{D P}$ and $\overline{T T D}$ are average delivery period and average time to delivery, respectively. The standard deviation of swap price returns are annualized (standard deviations are multiplied by the square root of 250 ).

DP. This observation suggest that the risk premium is increasing in volatility and rightskewed, which is in line with the hypotheses put forth in Bessembinder and Lemon [41]. They develop an equilibrium model for an electricity market with limited participation. Only players with physical capacity for electricity production or consumption are allowed to participate. In the Nord Pool financial market speculators are allowed as well, but the majority of market participants are still players with physical capacity. 
8.1.2. Descriptive statistics of price returns. Our main focus in the empirical part of the article is the dynamics of a family of lognormal models for the swap price dynamics, in particular the volatility dynamics. The models are calibrated to logreturns. Since a lognormal dynamics can not be assumed for overlapping contracts, these are removed from the sample. We remove contracts with long delivery periods, that completely overlap contracts with shorter delivery periods. For instance on March 3, 2004, referring to Table 1, 3 contracts are removed. FWYR-05 and ENOYR-06 cover the same delivery period as the seasonal contracts FWV1-05, FWS0-05, FWV1-05 and ENOQ1-06, ENOQ2-06, ENOQ306, ENOQ4-06 respectively, and they are therefore removed. FWS0-04 is also removed, since it completely overlaps the monthly contracts ENOMAY-04, ENOJUN-04, ENOJUL04, ENOAUG-04, ENOSEP-04. In total 1793 overlapping contracts were removed, leaving a total of 53919 price data.

Going from price level to logreturns one observation per contract is lost. Our total sample of approximately 8.5 years of daily data consists of 49425 logreturns. The number of non-overlapping contracts in our sample is 4494. Descriptive statistics of swap price returns are given in Table 4. From Panel A we see that the average daily return across all contracts is negative, suggesting overall negative drift for the swaps. The estimated standard deviation is multiplied by $\sqrt{250}$, since 250 is the average number of trading days in our sample. Average volatility is $50.2 \%$ for all contracts. We note the minimum return is $-66.0 \%$ and maximum return is $+69.0 \%$, giving no clear indication of skewness of returns. In Panel B we again sort the data in different sub-samples according the delivery period. Daily and weekly contracts have negative average returns, while monthly, seasonal and yearly have zero average returns down to three decimal accuracy.

Daily contracts with an average of 4 days to delivery have an estimated volatility of $111.6 \%$. Volatility for weekly contracts with is $54.7 \%$. For weekly, monthly, seasonal and yearly contracts volatility is $54.7 \% 38.9 \%, 21.7 \%, 18.2 \%$ with $\overline{T T D}=22,155,474$, and 538 , respectively.

Panel $\mathrm{C}$ sorts the data according to time to delivery; between two and six months, between six months and a year, and finally contracts with more than a year to delivery. The sub-sample of contracts with less than two months has negative average mean, while the other sub-samples have zero average means. Estimates of the standard deviations show an inverse relationship between volatility and TTD (and $D P$ ). This reinforces the results concluded from Panel B, where we showed the same inverse relationship between $D P$ and volatility. Since contracts with short $D P$ have short trading periods, they have low $\overline{T T D}$ and vice versa for contracts with long $D P$. This makes it hard to separate the effect of $D P$ and TTD simply by sorting the data. This will be clearer when we estimate models that accounts for both effects explicitly. The fact that volatility is decreasing in $D P$ and in $T T D$ is consistent with mean reversion in the underlying electricity price, in this case the day-ahead price. This pattern clearly indicates mean reversion in the underlying electricity 
price.Very high (low) electricity prices will result in very high (low) prices for a contract that is close to the delivery period. But mean reversion suggest that a very high (low) price today will be followed by a price decrease (increase) in the future. This way, contracts with long time to delivery will not increase (decrease) as much as short term contracts, since a price reversal is expected in the future.

Panel D sorts the data according to calendar time; one sub-sample for each quarter. Estimates of the standard deviations show some evidence of seasonal volatility. With TTD and $D P$ quite similar in all four quarters, the volatility effects due to these factors should also be quite similar across quarters. Still volatility is at its highest in the first quarter with the fourth quarter as the second highest volatility, $57.1 \%$ and $49.3 \%$, respectively. From April to September the average volatility is $46.35 \%$.

Next we proceed to specify and estimate a family of lognormal models.

8.2. Estimation of lognormal models and empirical results. In this sub-section we perform time series analysis on multiplicative diffusion models of the swap price dynamics. We assume that the dynamics of financial elctricity contracts traded at Nord Pool can be described by

$$
d F\left(t, T_{1}, T_{2}\right)=\lambda \Theta\left(t, T_{1}, T_{2}\right) F\left(t, T_{1}, T_{2}\right) d t+\Theta\left(t, T_{1}, T_{2}\right) F\left(t, T_{1}, T_{2}\right) d B(t)
$$

under the physical probability measure $P$, where $B(t)$ is a Brownian motion under $P$. The parameter $\lambda$ can be interpreted as the market price of risk. In all the models we discuss below, $\lambda$ is assumed to be constant. The function $\Theta\left(t, T_{1}, T_{2}\right)$ is deterministic, and in the empirical part in this study we disregard the jumps in the model. The log-returns are given by

$$
\begin{aligned}
\ln \left(\frac{F\left(t+\Delta t, T_{1}, T_{2}\right)}{F\left(t, T_{1}, T_{2}\right)}\right) & =\int_{t}^{t+\Delta t}\left(\lambda \Theta\left(s, T_{1}, T_{2}\right)-\frac{1}{2} \Theta^{2}\left(s, T_{1}, T_{2}\right)\right) d s \\
& +\int_{t}^{t+\Delta t} \Theta\left(s, T_{1}, T_{2}\right) d B(s)
\end{aligned}
$$

Setting $r\left(t, T_{1}, T_{2}\right):=\ln \left(\frac{F\left(t+\Delta t, T_{1}, T_{2}\right)}{F\left(t, T_{1}, T_{2}\right)}\right)$ we see that $r\left(t, T_{1}, T_{2}\right) \sim \mathcal{N}(m, v)$ where $\mathcal{N}(m, v)$ denotes a normally distributed random variable with mean

$$
m\left(t, T_{1}, T_{2}\right)=\int_{t}^{t+\Delta t}\left(\lambda \Theta\left(s, T_{1}, T_{2}\right)-\frac{1}{2} \Theta^{2}\left(s, T_{1}, T_{2}\right)\right) d s
$$

and variance

$$
v\left(t, T_{1}, T_{2}\right)=\int_{t}^{t+\Delta t} \Theta^{2}\left(s, T_{1}, T_{2}\right) d s
$$

For observation $i$ our empirical model is

$$
r^{i}\left(t, T_{1}, T_{2}\right)=m^{i}\left(t, T_{1}, T_{2}\right)+v^{i}\left(t, T_{1}, T_{2}\right) \varepsilon^{i}(t)
$$


where we assume $\varepsilon(t) \sim \mathcal{N}(0,1)$ and $m$ and $v$ are given above. The superscript $i$ is introduced to differentiate between returns on various contract on a given date $t$. There are on average 250 trading days a year, hence we set $\Delta t=1 / 250$. Rearranging the above expression we can write the error term as a function of the mean and variance of returns

$$
\varepsilon^{i}(t)=\frac{r^{i}\left(t, T_{1}, T_{2}\right)-m^{i}\left(t, T_{1}, T_{2}\right)}{v^{i}\left(t, T_{1}, T_{2}\right)}
$$

Let $\phi$ be the parameters to be estimated and $\Phi$ the parameter space. The likelihood for observation $\varepsilon^{i}(t)$ at time $t$, considered as a function of $\phi$ (and $t, T_{1}$ and $T_{2}$ ) is denoted $L^{i}\left(\phi, t, T_{1}, T_{2}\right)$. Let $l^{i}\left(\phi, t, T_{1}, T_{2}\right)$ be the log-likelihood of $L^{i}\left(\phi, t, T_{1}, T_{2}\right)$. With $N$ observations the log-likelihood function becomes

$$
l\left(\phi, t, T_{1}, T_{2}\right)=\sum_{i=1}^{N} l^{i}\left(\phi, t, T_{1}, T_{2}\right)
$$

were $l$ is the standard normal density functions. $\widehat{\phi}_{M L}$ is the maximum likelihood estimator of $\phi$ if $l(\widehat{\phi}) \geq l(\phi) \forall \phi \in \Phi$. Several of the parameters are assumed to be positive. We handle this using a 2 step procedure: First re-parametrize the parameters so that they cannot become negative. Then estimate these re-parametrized parameters using standard unconstrained optimization. Suppose we are interested in estimating one parameter only, $\phi=\phi_{1}$, and that this parameter is non-negative by assumption. We then define the re-parametrized parameter as $\bar{\phi}=\bar{\phi}_{1}$. The actual reparametrization ${ }^{9}$ we use is

$$
\bar{\phi}_{1}=U \frac{e^{-\phi_{1}}}{1+e^{-\phi_{1}}}
$$

This ensures that $\phi_{1} \in[0, U]$. If the only requirement is positivity, $U$ is set at a very high value. Standard, unconstrained, optimization can now be performed on $l\left(\bar{\phi}, t, T_{1}, T_{2}\right)$. Finally we can calculate $\widehat{\phi}_{M L}$ from $\widehat{\bar{\phi}}_{M L}$ using (8.3). In case of misspecification we apply the method suggested by White [57] to compute parameter standard deviations. Let $J_{\widehat{\phi}_{M L}}$ and $H_{\widehat{\phi}_{M L}}$ denote the Jacobian and Hessian matrix of the likelihood functions. The White heteroscedastic consistent covariance matrix is given by

$$
\left(H_{\widehat{\phi}_{M L}}\right)^{-1}\left(J_{\widehat{\phi}_{M L}^{\top}}^{\top} J_{\widehat{\phi}_{M L}}\right)\left(H_{\widehat{\phi}_{M L}}\right)^{-1}
$$

were $A^{-1}$ and $A^{\top}$ mean inverse and transpose, respectively, of a matrix $A$. Both $J_{\widehat{\phi}_{M L}}$ and $H_{\widehat{\phi}_{M L}}$ are estimated numerically.

Turning to the specification of $\Theta$, we investigate all six volatility functions summarized in Table 2. Parameter estimates for models E1-E6 are given in Table 5. The parameter $a$ in model E1 recaptures the annual volatility averaged over all contracts already documented in Panel B of Table 4. Introducing exponential decaying volatility in model E2 improves the

\footnotetext{
${ }^{9}$ This method is suggested by Harvey [25].
} 


\begin{tabular}{ccccccc} 
Parms. & E1 & E2 & E3 & E4 & E5 & E6 \\
\hline$\lambda$ & -0.187 & -0.149 & -0.149 & -0.093 & 0.023 & 0.016 \\
& $(0.048)$ & $(0.005)$ & $(0.011)$ & $(0.015)$ & $(0.007)$ & $(0.002)$ \\
$a$ & 0.502 & 0.634 & 1.178 & 0.856 & 0.827 & 0.619 \\
& $(0.009)$ & $(0.012)$ & $(0.009)$ & $(0.025)$ & $(0.021)$ & $(0.018)$ \\
$b$ & & 0.629 & 0.629 & 2.424 & 2.777 & 3.007 \\
& & $(0.015)$ & $(0.014)$ & $(0.249)$ & $(0.249)$ & $(0.221)$ \\
$c$ & & & & 0.293 & 0.205 & 0.183 \\
& & & & $(0.024)$ & $(0.011)$ & $(0.008)$ \\
$d$ & & & -0.007 & & 0.130 & 0.043 \\
& & & $(0.000)$ & & $(0.021)$ & $(0.006)$ \\
$f$ & & & 0.543 & & -0.202 & -0.081 \\
& & & $(0.003)$ & & $(0.014)$ & $(0.006)$ \\
\hline LL & 100401.8 & 111249.6 & 111249.6 & 113559.2 & 115701.7 & 116229.4
\end{tabular}

TABLE 5. Parameter estimates for models with exponentially decaying volatility. The table shows parameter estimates from maximum-likelihood estimation on electricity swap contracts traded on Nordpool. Estimations are made separately on daily observations of all contracts in the period 1989-1999 using a toal of 42858 observations. The specification of model E1-E6 is given in Table 2. We also report the maximised log-likelihood function (LL) and heteroscedasticity consistent standard deviation (White [57]) in parantheses. All parameters are significant at $1 \%$ level of significance.

log-likelihood function. The parameter $a$ increases compared to model E1 which means that implied spot volatilty is $63.4 \%$. The decay factor $b$, with an estimate of 0.629 , suggests a fairly modest decrease in volatility as time to maturity increases. This estimate reflects the compromise between very high volatility for short term contracts, and considerably lower volatility for longer contracts evident from Table 4. Model E3 introduces deterministic seasonal volatility. Judging by the log-likelihood function, this modification does not fit the data better than the more parsimoneous model E2. We also note that the parameter estimate for $b$ is unchanged. The parameters for $a, d$ and $f$ provides a large degree of flexibility in determining spot volatility, and very different combination of parameters produce very similiar results in terms of data fit. In fact, we experimented with different starting values, and this always gave more or less identical values of the parameter $b$, different parameter values for $a, d$ and $f$, and identical value of the log-likelihood function. The evidence so far suggest that the maturity effect is present in the data and seasonality is not. However, in E3 the maturity effect modeled by a simple negative exponential 
A) Implied time dependent volatility for each contract

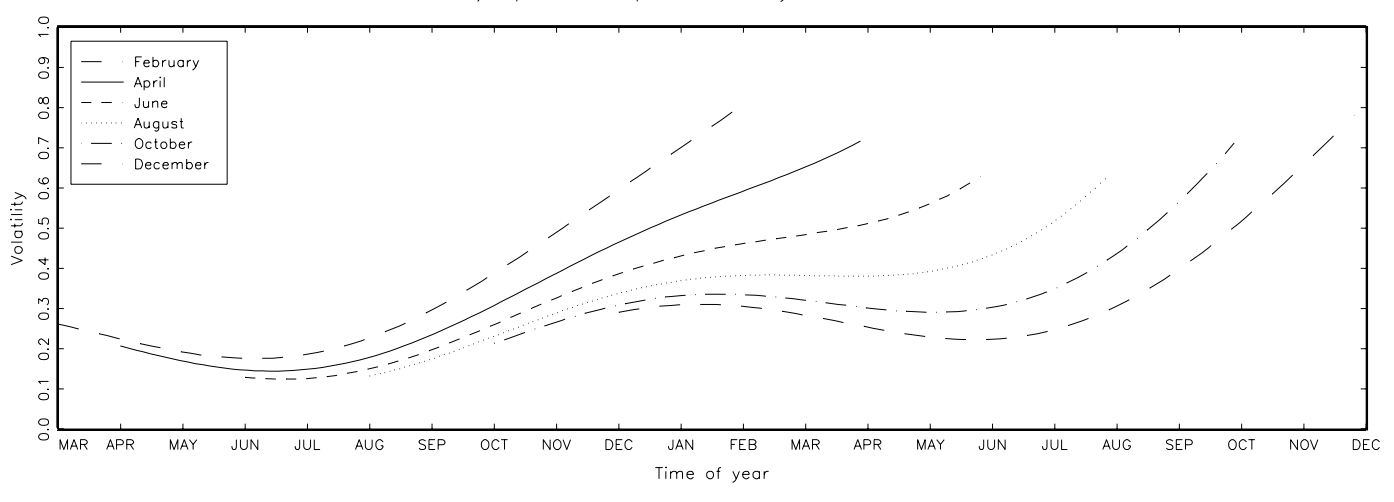

B) Maturity effect

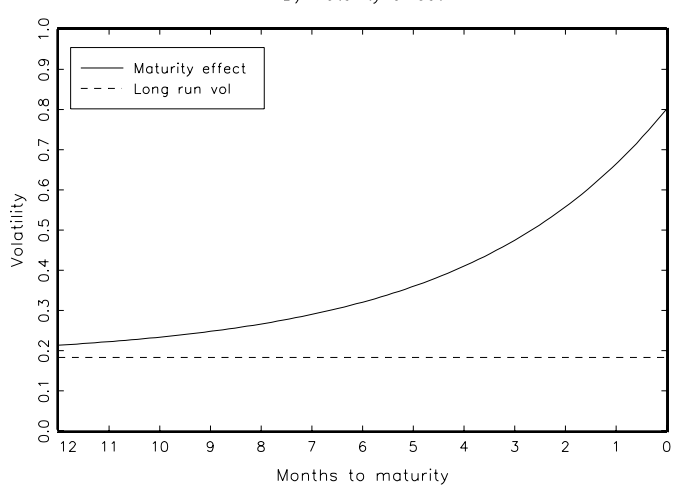

C) Implied seasonal spot price vol

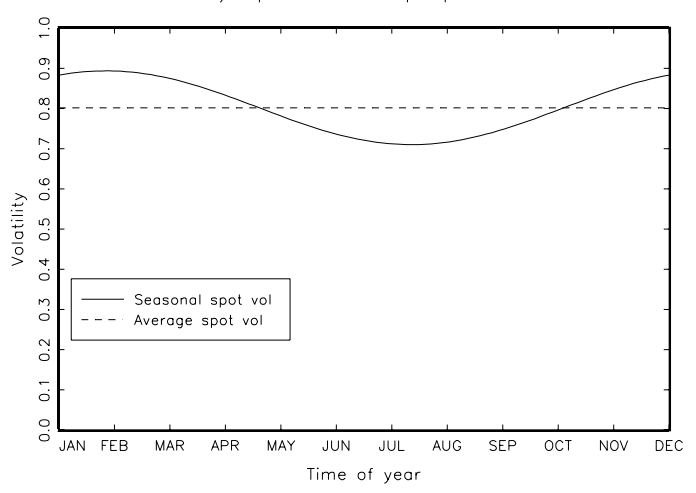

FiguRE 3. Maturity and seasonal effect and the combined effect on the volatility dynamics. The volatility function is E6 in Table 2 and parameters are in Table 5.

function, implying that long run volatility approaches zero. Furthermore, the maturityand the seasonality functions are multiplicative. It turns out, modifying both these features gives a different conclusion.

Model E4 adds flexibility in the structure of decaying volatility. We note that this model fits the data better than model E2 and E3. The estimate for parameter $a$ has increased to $85.6 \%$. This shows that this model better captures the high volatility for contracts with short time to maturity as is evident from Table 4 . The estimate for $b$ is now much higher compared to model E2 and E3, suggesting quick decay in volatility in time to maturity. The long run volatility in this model is given by $a \times b$. The parameters of model E4 imply approximately $21 \%$ long run volatility. This number seems a bit high compared to the annulised standard deviation of the sub-sample of returns on yearly contracts in Table 4 .

Model E5 and E6 keeps the flexibility of the decaying volatility from E4 and adds seasonality. In E5 the seasonal- and maturity effect is multiplicative, and in the volatility function of E6 the two effects are additive. For model E5 the estimates of $a, b$ and $c$ are 


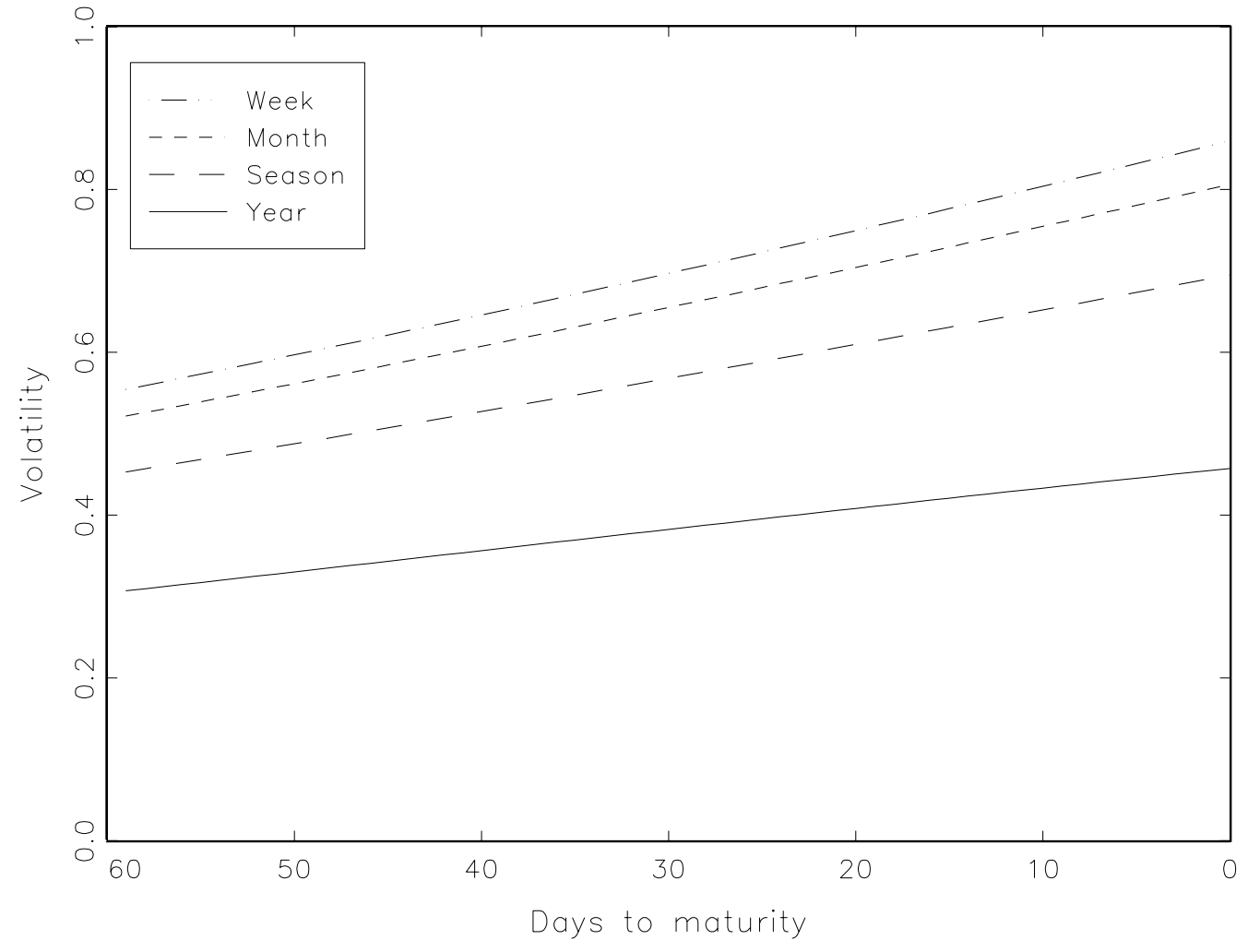

Figure 4. Time dependent volatility for contracts with common time to delivery and different delivery periods. The volatility function is E6 in Table 2 and parameters are in Table 5.

very similiar to model E4. But this model specification did not lead to any problems with estimation. In this model all the parameters converge, including the ones governing seasonality. Note the substantial increase in log-likelihood function compared to model E4 without seasonal volatility. Model E6 provides best fit to data. In E6 the implied spot volatility is approximately $80 \%(a+c)$. Long term volatility, ignoring seasonality is $18.3 \%$ (parameter value $c$ ). This is very close to average volatility of the yearly contracts in Table 4. Also note that the parameter value $b$ is the highest for E6 compared to the other models, meaning that this model gives the most profound maturity effect. The main insights from this class of models are that the maturity effect is a very important feature of the data. Modeling this with a simple exponential function is inadequate. There is also evidence of seasonal volatility, and our estimation results indicate that these two effects are best captured using an additive volatility function.

In Figures 3 and 4 we visualize how the maturity- and seasonality effect are picked up in the traded contracts. In both these figures we have used the swap volatility function E6 
and parameter values from Table 5. In the lower right panel of Figure 3 we have "turned off" the maturity effect, and plotted spot volatility $(a+c(t))$. We see that average implied spot volatility is just above $80 \%$ (dashed line), and that seasonality implies a winter peak of approximately $90 \%$ in February-March and low of $70 \%$ in mid-summer. Note that our seasonality specification is completely symmetrical. The peak follows 6 months after the low, and high winter volatility in turn forces the summer volatility to be equally low. This might be a too restrictive model of seasonality. We will comment on this when we investigate the residuals from this model below. Panel B of Figure 3 shows the implied forward curve for model E6 with seasonality turned off (setting $d=f=0$ ). So ignoring seasonality we see that volatility rises sharply as maturity approaches. The dashed line is the long run volatility of $18.3 \%$ (parameter value of the constant $c$ ). Note that the strong maturity effect in this market increase implied forward curve volatility from approximately $20 \%$ to $80 \%$ during the last 12 months prior to expiration. We have argued that neither fixed maturity forwards nor pure spot prices exist in this market, so the top panel shows how these effects are combined in the swap volatility for traded contracts. We have plotted volatility functions for 6 monthly contracts each being alive for 1 year. Following each contract for such a long period of time clearly shows the impact of both the seasonaland maturity effect on each contract. For the winter contracts seasonality reinforces the maturity effect, and for the summer contracts seasonality dampens it.

In Figure 4 we illustrate how the length of the delivery period affects volatility. Here we have plotted the time dependent volatility for contracts with delivery period of one week, month, quarter and year. The starting date of the delivery period for the contracts is set to January 1. Volatility increases steadily for all contracts the last 60 days prior to delivery, steepest increase for the contracts with the shortest delivery period. Seasonality affects the level of volatility for all contracts, but it is not visible when we plot the volatility functions only the last two months prior to maturity.

8.3. Analysing normalised returns. We have proposed a very simple model for the price movements for the electricty contracts on Nord Pool. According to (8.1) returns are normally distributed. We want to investigate the improvement of goodness of fit from our simplest model E1 to the one which gave the best fit E6. That is, we compute

$$
\widehat{\varepsilon}_{j}^{i}(t)=\frac{w^{i}\left(t, T_{1}, T_{2}\right)-m_{j}^{i}\left(t, T_{1}, T_{2}\right)}{v_{j}^{i}\left(t, T_{1}, T_{2}\right)}
$$

were $\widehat{\varepsilon}_{j}^{i}(t)$ is estimated residual for the return on contract $i$ for model $j$. Model E1 is indicated by $j=1$ and $j=6$ is model E6. We use our parameter estimates for the model E1 and E6 in Table 5 to compute $m$ and $v$. For a correctly specified model the normalised returns should be normally distributed with zero mean and standard deviation of one. Skewness and excess kurtosis should be zero. In Table 6 we present unconditional estimates 
of mean, standard deviation, skewness and excess kurtosis for standardised returns for model E1 and E6. Note that model E1 implies constant variance and mean for the returns on all contracts. From panel A we see that unconditional mean and standard deviation are equal to 0 and 1, respectively. This is not surprising, since this is what we have instructed the maximum likelihood procedure to do. Model E6 implies higher positive skewness than E1. The most prominent feature of the data is kurtosis. Since E1 neglects possible time-dependent volatility, it is not surprising that this model specification produce more leptokurtic standardised returns than model E6.

In Panel $\mathrm{B}$ we sort the data according to the length of the delivery period, looking at daily-, weekly-, monthly, seasonal- and yearly contracts individually. The estimates for the unconditional mean is qualitatively equal for model E1 and E6. This is somwhat surprising since the parameter estimates in Table 5 suggest very different estimates for the mean of the models. We see that the market price of risk in E1 is negative, suggesting overall negative risk premium in this market for model E1. For model E6 we draw the opposite conclusion. Still, the normalised returns of daily and weekly contracts have a negative mean which is unaccounted for by both models. The oppsosite is true for monthly, seasonal and yearly contracts. This suggest that the risk premium is not equal across contracts. Model E1 underestimates the standard deviation of daily- and weekly contracts, and overestimates the others. This is not surprising, due to the constant volatility assumption, and the clear indication of time dependent volatility in this market. Model E6 underestimates the volatility of the daily contracts and overestimates the volatility of the weekly contracts. This suggest that the a single exponential function might not quite capture the maturity effect, especially for contracts with short delivery period. We see from the numbers in the table that our volatility function does not quite capture this very significant drop from the daily to the weekly contracts. For monthly and seasonal contracts, the estimates produce volatility very close to empirical ones, and for yearly contracts the model estimate is above the empirical estimate. Model E6 has skewness and excess kurtosis estimates closer to zero than E1 in each sub-sample, but they are still far from zero especially for the kurtosis. It is interesting to note that for the monthly and seasonal contracts, where E6 seem to make a very good job at capturing the standard deviation of empirical returns, the excess kurtosis is less than half that of a model with contant volatility. This is very clear evidence that the maturity effect picks up a very important feature of the data.

In Panels $\mathrm{C}$ and $\mathrm{D}$ we sort the data according to time to delivery and time of year respectively. In Panel $\mathrm{C}$ the sub-sample of contracts with less than two months to delivery is dominated by contracts with short delivery periods (daily- and weekly contracts), and the sub-sample of contracts with delivery more than year ahead consists entirely of seasonal and yearly contracts. So the results in Panel $\mathrm{C}$ are somewhat comparable to Panel $\mathrm{B}$. We note that the mean is negative only for the first sub-sample and positive for the others. This suggest that the risk premium might change from positive to negative during the life 


$$
\begin{aligned}
& \text { Mean Stand. dev. Skewness Ex. kurtosis } \\
& \begin{array}{llllllll}
\widehat{\varepsilon}_{1} & \widehat{\varepsilon}_{6} & \widehat{\varepsilon}_{1} & \widehat{\varepsilon}_{6} & \widehat{\varepsilon}_{1} & \widehat{\varepsilon}_{6} & \widehat{\varepsilon}_{1} & \widehat{\varepsilon}_{6}
\end{array}
\end{aligned}
$$

Panel A: Full sample

\begin{tabular}{lllllllll}
\hline All & 0.00 & 0.00 & 1.00 & 1.00 & 0.65 & 1.17 & 62.48 & 40.92
\end{tabular}

Panel B: Sub-samples sorted according to DP

\begin{tabular}{lrrrrrrrr}
\hline Day & -0.14 & -0.08 & 2.22 & 1.42 & 0.21 & 0.19 & 15.80 & 15.91 \\
Week & -0.03 & -0.01 & 1.09 & 0.79 & -0.10 & -0.09 & 17.25 & 14.24 \\
Month & 0.03 & 0.01 & 0.77 & 1.01 & 3.56 & 1.95 & 116.45 & 49.69 \\
Season & 0.03 & 0.02 & 0.43 & 1.00 & 2.76 & 1.46 & 120.62 & 50.79 \\
Year & 0.03 & 0.02 & 0.36 & 0.75 & 1.76 & 0.50 & 40.79 & 8.28
\end{tabular}

Panel C: Sub-samples sorted according to TTD

\begin{tabular}{lrrrrrrrr}
\hline$[0,2$ mon. $\rangle$ & -0.05 & -0.03 & 1.44 & 0.99 & 0.24 & 0.40 & 30.65 & 28.70 \\
{$[2$ mon., 6 mon. $)$} & 0.03 & 0.02 & 0.80 & 0.99 & 4.13 & 3.21 & 118.30 & 72.35 \\
[6 mon., 1 year $\rangle$ & 0.02 & 0.01 & 0.59 & 1.09 & 0.81 & 0.80 & 43.20 & 30.43 \\
{$[1$ year, $\rightarrow\rangle$} & 0.03 & 0.01 & 0.37 & 0.95 & 2.25 & 1.10 & 127.76 & 46.29
\end{tabular}

Panel D: Sub-samples sorted according to time of year

\begin{tabular}{lrrrrrrrr}
\hline 1. quarter & -0.05 & -0.05 & 1.14 & 1.07 & 2.31 & 3.45 & 77.31 & 87.11 \\
2. quarter & 0.02 & 0.04 & 0.92 & 0.95 & -2.30 & -0.15 & 37.54 & 13.94 \\
3. quarter & 0.01 & 0.01 & 0.93 & 1.00 & 0.76 & 0.28 & 76.01 & 16.22 \\
4. quarter & 0.02 & 0.00 & 0.98 & 0.97 & 0.23 & 0.15 & 36.95 & 20.08
\end{tabular}

TABLE 6. Normalised residuals devation from standard normal distribution. The columns $\widehat{\varepsilon}_{1}$ and $\widehat{\varepsilon}_{6}$ represent normalised residuals from model E1 and E6 using the parameter estimates from Table 5.

of a contract. However, turning to Panel D we note that normalised returns have negative means only on contracts traded in the first three months of the year. This is an indication of seasonal risk premium, and it is true for both models. In future research focusing on the risk premium in this market, one should consider investigating the risk premium as a function of seasonality, time to delivery and the length of the delivery period. Model E6 actually assumes risk premium that varies according to season, time to delivery and delivery period, since the total risk premium is a function of constant market price of risk and a volatility function that depends on $t, T_{1}$ and $T_{2}$. Model E1 on the other hand 

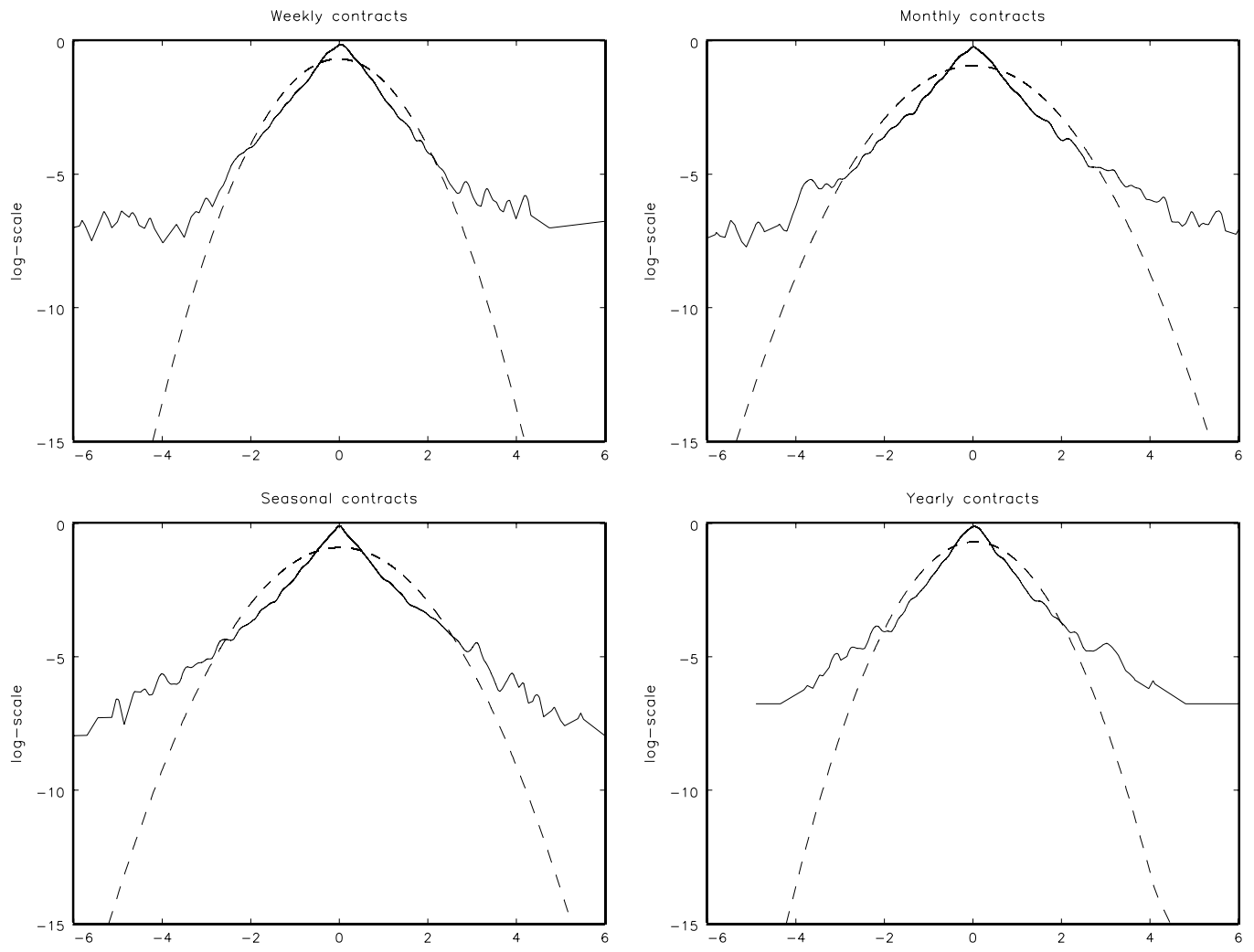

FiguRE 5. Density plots of normalised returns for contracts of different delivery period. The dashed line shows the normal distribution with the estimatwed mean and standard deviation for the normalised returns for comparison. Vertical axes are on log-scale.

assumes constant risk premia for all contracts at all times. We note that the normalised returns for both models have qualitatively similiar results for the mean in all panels of Table 6 suggesting that neither model is able to capture the variations in the risk premium satisfactorily.

Looking at the estimates for skewness and excess kurtosis in Panels C and D we find again that normalised residuals for both models are far from standard normal, but residuals from model E6 are closer to normality than model E1. The exception is the 1. quarter sub-sample in Panel D. We see that the estimates of both skewness and kurtosis are higher for model E6 than the E1. The fact that model E6 does not fit considerably better than model E1 indicates that the seasonal specification of the model can be improved.

Table 6 indicates that time varying volatility is an important feature of the data. It also suggest that using a simple trigonometric function to capture seasonality, and a modified exponential function to capture the maturity effect, as is done in model E6, can probably 
be improved to give an even better fit to the data. However, the main result from Table 6 is that the normalised returns are far from being normally distributed. On average we see that normalised returns are skewed to the right, with very high excess kurtosis. In Figure 5 we provide empirical density plots for the normalised returns for sub-samples of weekly-, monthly-, seasonal-, and yearly contracts. The dashed lines are the normal density with mean and standard deviation corresponding to each sub-sample. We present the plots with vertical axes on log-scale to better visualise the probability mass in the tails of the empirical distributions. The high estimates of kurtosis from Table 6 result in very heavy tails. This suggest that a model for the logreturns might be substantially improved by fitting it with a different density than the normal, as we have done in this paper. The plots show a tent-shaped distribution having similar characteristics as produced by the generalized hyperbolic distributions (see e.g. Barndorff-Nielsen and Shephard [3]). It is a very challenging task to model the residuals, since all the moments of the distribution might potentially depend on $t, T_{1}$ and $T_{2}$.

8.4. Further empirical research. Our lognormal model is very simple using only one Brownian motion to describe the swap price movements. However, as we have discussed thoroughly in the previous sections, it is able to pick up quite a lot of the stylised facts in this market; most notably the seasonality and maturity effect of the swap volatility.

In order to improve the model for swap price dynamics, we could allow for more than one Wiener component in the model. This is a straightforward task in the theoretical description of the stochastic dynamics, and basically a matter of notation. But the flexibility in the specification of different volatility effects is improved, and may include two factor models like the one suggested by Kellerhals [34], which has a very strong correlation between contracts with different time to maturity and delivery periods. In an empirical paper analysing Nord Pool data, Koekebakker and Ollmar [38] show that this correlation is weaker in the electricity market compared to many other commodity markets. A more explicit modeling of correlation, like the one proposed in Keppo et al. [35], is another potential extension of this research.

Since our theoretical model allows for jumps in swap prices, one very interesting extension could be to test out different classes of distributions like the generalized hyperbolic one to describe the heavy tails in price returns. Such a specification will give rise to a Lévy process in the dynamics. Furthermore, Geman and Roncoroni [23] have suggested a very interesting jump-diffusion model for spot electricity, that perhaps can be applicable also in a term structure model.

\section{Conclusions}

In this article we have provided a continuous time mathematical framework for swap curve modeling in electricity markets. Since financial electricity contracts are specified 
with a delivery period, they are financially speaking swaps. Furthermore, since these swaps are the main hedging tools in this industry, the dynamics of these contracts of interest for market participants.

Within our theoretical model we investigate the relationship between spot-, forward curve (fixed delivery forwards) and swap price dynamics. We provide no-arbitrage relationships between these entities and propose two approaches to model the swap dynamics based on the HJM-approach. All models we consider allow for a Wiener and a jump part in their dynamics. By modeling the non-traded fixed-delivery forwards, we end up with a rather complicated dynamics for the swap price, which may in general be too cumbersome for practical implementation of risk management tools and option pricing. On the other hand, if we model the swap dynamics directly for all theoretically possible delivery times, we are led to complicated conditions on the parameters of the process, which for instance is not satisfied for general lognormal models. Hence, we end up with proposing a simplified version of the HJM-approach, only modeling those swaps which are actually traded. We show that this leads to explicit option pricing formulas when using a lognormal specification, and hedging parameters can be explicitly calculated.

In the empricial part of our paper we suggest a simple one-factor lognormal model for swap price dynamics, and estimate several specifications of the volatility structure using the principle of maximum likelihood estimation on data collected from the Nordic power exchange Nord Pool. Our main empirical results are as follows: We find a very strong maturity effect. This cannot be picked up by a simple negative exponential function. We also find evidence of seasonally changing volatility. Finally, we observe that the lognormal model is unable to explain the heavy tails of logreturns. This leaves plenty of room for future research.

\section{Appendix A. TeChnical COnditions}

We assume the following conditions on the dynamics for the swap, forward and spot in (3.1) -(3.3), respectively:

Condition $(\boldsymbol{S} \boldsymbol{W})$ : For the dynamics in (3.1), assume that the following hold:

- The random field $\left(t, T_{1}, T_{2}, \omega\right) \mapsto \Sigma\left(t, T_{1}, T_{2}, \omega\right)$ is $\mathcal{B} \times \mathcal{F}$-measurable, where $\mathcal{B}$ is the Borel $\sigma$-algebra on $\left\{\left(t, T_{1}, T_{2}\right) \in[0, T]^{3}: t \leq T_{1}<T_{2}\right\}$

- The random field $\left(t, T_{1}, T_{2}, z, \omega\right) \mapsto \Psi\left(t, T_{1}, T_{2}, z, \omega\right)$ is $\mathcal{B} \times \mathcal{F}$-measurable, where $\mathcal{B}$ is the Borel $\sigma$-algebra on $\left\{\left(t, T_{1}, T_{2}, z\right) \in[0, T]^{3} \times \mathbb{R}: t \leq T_{1}<T_{2}\right\}$.

- For all $\left(T_{1}, T_{2}\right)$ such that $0<T_{1} \leq T_{2} \leq T,\left[0, T_{1}\right] \ni t \mapsto \Sigma\left(t, T_{1}, T_{2}\right)$ is progressively measurable, $\left[0, T_{1}\right] \times \mathbb{R} \ni(t, z) \mapsto \Psi\left(t, T_{1}, T_{2}, z\right)$ is a predictable process (in the sense of Ikeda and Watanabe, [31, Def. 3.3, p. 61]) and

$$
\mathbb{E}\left[\int_{0}^{T_{1}} \Sigma^{2}\left(t, T_{1}, T_{2}\right) d t\right]<\infty, \mathbb{E}\left[\int_{0}^{T_{1}} \int_{\mathbb{R}} \Psi^{2}\left(t, T_{1}, T_{2}, z\right) \ell(d z) d t\right]<\infty .
$$


Condition $(\boldsymbol{F})$ : For the dynamics in (3.2), assume that following hold:

- The random field $(t, u, \omega) \mapsto \sigma(t, u, \omega)$ is $\mathcal{B} \times \mathcal{F}$-measurable, where $\mathcal{B}$ is the Borel $\sigma$-algebra on $\left\{(t, u) \in[0, T]^{2}: t \leq u\right\}$

- The random field $(t, u, z, \omega) \mapsto \psi(t, u, z, \omega)$ is $\mathcal{B} \times \mathcal{F}$-measurable, where $\mathcal{B}$ is the Borel $\sigma$-algebra on $\left\{(t, u, z) \in[0, T]^{2} \times \mathbb{R}: t \leq u\right\}$.

- For all $0<u<T,[0, u] \ni s \mapsto \sigma(s, u)$ is progressively measurable, $[0, u] \times \mathbb{R} \ni$ $(s, z) \mapsto \psi(s, u, z)$ is a predictable process (in the sense of Ikeda and Watanabe, $[31$, Def. 3.3, p. 61]) and

$$
\mathbb{E}\left[\int_{0}^{u} \sigma^{2}(s, u) d s\right]<\infty, \mathbb{E}\left[\int_{0}^{u} \int_{\mathbb{R}} \psi^{2}(s, u, z) \ell(d z) d s\right]<\infty .
$$

Condition ( $\boldsymbol{S})$ : For the dynamics in (3.3), assume that the following hold:

- $\alpha$ and $\beta$ are progressively measurable stochastic processes which satisfy the integrability conditions

$$
\mathbb{E}\left[\int_{0}^{T} \alpha^{2}(t) d t\right]<\infty, \mathbb{E}\left[\int_{0}^{T} \beta^{2}(t) d t\right]<\infty .
$$

- $\gamma(t, z)$ is a predictable process (in the sense of Ikeda and Watanabe, [31, Def. 3.3, p. 61]) with the property

$$
\mathbb{E}\left[\int_{0}^{T} \int_{\mathbb{R}} \gamma^{2}(t, z) \ell(d z) d t\right]<\infty .
$$

To state further conditions on the coefficient functions, introduce the following notation: let $\mathcal{B}(A)$ be the Borel $\sigma$-algebra on a topological space $A$, and $\mathcal{S}$ the predictable $\sigma$-algebra in the sense of Ikeda and Watanabe, [31, Def. 3.3, p. 61]. Introduce the notation $\partial_{i}$ for $i=1,2,3$ for partial differentiation with respect to the $i$ th variable of a function.

To connect the forward dynamics with the spot price, we introduce Condition (F-S):

Condition $(\boldsymbol{F}-\boldsymbol{S})$ : For the dynamics in (3.2) and (3.3), suppose the following (in addition to Conditions $(\mathbf{F})$ and $(\mathbf{S}))$ :

- $\partial_{2} \sigma$ and $\partial_{2} \psi$ exist

- $[0, T] \times \Omega \times[0, T] \ni((s, \omega), u) \mapsto 1_{[s, T]}(u) \partial_{2} \sigma(s, u, \omega)$ is jointly progressively measurable and measurable with respect to $\mathcal{B}([0, T])$,

- $(u, \omega) \mapsto 1_{[s, T]}(u) \partial_{2} \sigma(s, u, \omega)$ is $\mathcal{B}([0, T]) \times \mathcal{F}$-measurable.

- $[0, T] \times \Omega \times[0, T] \times \mathbb{R} \times \ni((s, \omega), u, z) \mapsto 1_{[s, T]}(u) \partial_{2} \psi(s, u, z, \omega)$ is $\mathcal{S} \times \mathcal{B}([0, T])$ measurable,

- $(z, u, \omega) \mapsto 1_{[s, T]}(u) \partial_{2} \psi(s, u, z, \omega)$ is $\mathcal{B}(\mathbb{R}) \times \mathcal{B}([0, T]) \times \mathcal{F}$-measurable for every $s$. 
- The following integrability holds

(A.5)

$\mathbb{E}\left[\int_{0}^{T} \int_{0}^{T} 1_{[s, T]}\left(\partial_{2} \sigma(s, u)\right)^{2} d s\right]<\infty, \mathbb{E}\left[\int_{0}^{T} \int_{0}^{T} \int_{\mathbb{R}} 1_{[s, T]}\left(\partial_{2} \psi(s, u, z)\right)^{2} \ell(d z) d s d u\right]<\infty$.

- $s \mapsto \sigma(s, s)$ is progressively measurable, $(s, z) \mapsto \psi(s, s, z)$ predictable, and

$$
\begin{aligned}
& \mathbb{E}\left[\int_{0}^{T} \sigma^{2}(s, s) d s\right]<\infty \\
& \mathbb{E}\left[\int_{0}^{T} \int_{\mathbb{R}} \psi^{2}(s, s, z) \ell(d z) d s\right]<\infty .
\end{aligned}
$$

- The initial forward curve $[0, T] \ni t \mapsto f(0, t)$ is differentiable with respect to its second variable, $\partial_{2} f(0, t)$ is $\mathcal{B}([0, T])$-measurable, and

$$
\int_{0}^{T}\left(\partial_{2} f(0, t)\right)^{2} d t<\infty
$$

To connect the swap dynamics with the forward price, we introduce some additional conditions on the coefficients in the forward dynamics which we refer to as Condition (F-SW):

Condition $(\boldsymbol{F}-\boldsymbol{S} \boldsymbol{W})$ : For the dynamics in (3.2) and (3.1), suppose the following (in addition to Conditions $(\mathbf{F})$ and $(\mathbf{S W}))$ :

- $\left[0, T_{1}\right] \times \Omega \times\left[T_{1}, T_{2}\right] \ni((s, \omega), u) \mapsto \widehat{w}\left(u ; T_{1}, T_{2}\right) \sigma(s, u, \omega)$ is jointly progressively measurable and measurable with respect to $\mathcal{B}\left(\left[T_{1}, T_{2}\right]\right)$,

- $(u, \omega) \mapsto \widehat{w}\left(u ; T_{1}, T_{2}\right) \sigma(s, u, \omega)$ is $\mathcal{B}\left(\left[0, T_{1}\right]\right) \times \mathcal{F}$-measurable,

- $\left[0, T_{1}\right] \times \Omega \times\left[T_{1}, T_{2}\right] \times \mathbb{R} \times \ni((s, \omega), u, z) \mapsto \widehat{w}\left(u ; T_{1}, T_{2}\right) \psi(s, u, z, \omega)$ is $\mathcal{S} \times \mathcal{B}\left(\left[T_{1}, T_{2}\right]\right)$ measurable,

- $(z, u, \omega) \mapsto \widehat{w}\left(u ; T_{1}, T_{2}\right) \psi(s, u, z, \omega)$ is $\mathcal{B}(\mathbb{R}) \times \mathcal{B}\left(\left[T_{1}, T_{2}\right]\right) \times \mathcal{F}$-measurable for every $s \leq T_{1}$.

- The following integrability holds

$$
\begin{aligned}
& \mathbb{E}\left[\int_{0}^{T_{1}} \int_{T_{1}}^{T_{2}} \widehat{w}^{2}\left(u ; T_{1}, T_{2}\right) \sigma^{2}(s, u) d s\right]<\infty, \\
& \mathbb{E}\left[\int_{0}^{T_{1}} \int_{T_{1}}^{T_{2}} \int_{\mathbb{R}} \widehat{w}^{2}\left(u ; T_{1}, T_{2}\right) \psi^{2}(s, u, z) \ell(d z) d s d u\right]<\infty .
\end{aligned}
$$

- The initial forward curve $\left[T_{1}, T_{2}\right] \ni t \mapsto f(0, t) \mathcal{B}\left(\left[T_{1}, T_{2}\right]\right)$-measurable and

$$
\int_{T_{1}}^{T_{2}} \widehat{w}^{2}\left(t ; T_{1}, T_{2}\right) f^{2}(0, t) d t<\infty
$$




\section{Appendix B. Proofs}

Proof of Lemma 4.1. Dividing by $T_{2}-T_{1}$ in the numerator and denominator, and letting $T_{2} \downarrow T_{1}$ leads to

$$
\lim _{T_{2} \downarrow T_{1}} F\left(t, T_{1}, T_{2}\right)=\frac{\left.\left(\frac{\partial}{\partial T_{2}} \int_{T_{1}}^{T_{2}} w(u) f(t, u) d u\right)\right|_{T_{2}=T_{1}}}{\left.\left(\frac{\partial}{\partial T_{2}} \int_{T_{1}}^{T_{2}} w(u) d u\right)\right|_{T_{2}=T_{1}}}=f\left(t, T_{1}\right), \text { a.e.t } \in\left[0, T_{1}\right],
$$

by the Fundamental Theorem of Calculus. This proves the Lemma.

Proof of Lemma 4.2. Consider first $N=3$. A straightforward calculation reveals

$$
\begin{aligned}
F\left(t, T_{1}, T_{3}\right) & =\int_{T_{1}}^{T_{2}} \widehat{w}\left(t ; T_{1}, T_{3}\right) f(t, u) d u+\int_{T_{2}}^{T_{3}} \widehat{w}\left(t ; T_{1}, T_{3}\right) f(t, u) d u \\
& =\frac{\int_{T_{1}}^{T_{2}} w(u) d u}{\int_{T_{1}}^{T_{3}} w(u) d u} F\left(t, T_{1}, T_{2}\right)+\frac{\int_{T_{2}}^{T_{3}} w(u) d u}{\int_{T_{1}}^{T_{3}} w(u) d u} F\left(t, T_{2}, T_{3}\right)
\end{aligned}
$$

which is the desired relation for $N=3$. The result holds for general $N$ by induction.

Proof of Theorem 4.3. From the link (4.1) and Condition (F-SW) we find after appealing to the stochastic Fubini theorem $\left(t \leq T_{1}\right)$

$$
\begin{aligned}
F\left(t ; T_{1}, T_{2}\right)= & \int_{T_{1}}^{T_{2}} \widehat{w}\left(u ; T_{1}, T_{2}\right) f(0, u) d u+\int_{T_{1}}^{T_{2}} \int_{0}^{t} \widehat{w}\left(u ; T_{1}, T_{2}\right) \sigma(s, u) d W(s) d u \\
& \quad+\int_{T_{1}}^{T_{2}} \int_{0}^{t} \int_{\mathbb{R}} \widehat{w}\left(u ; T_{1}, T_{2}\right) \psi(s, u, z) \widetilde{N}(d z, d s) d u \\
= & \int_{T_{1}}^{T_{2}} \widehat{w}\left(u ; T_{1}, T_{2}\right) f(0, u) d u+\int_{0}^{t} \int_{T_{1}}^{T_{2}} \widehat{w}\left(u ; T_{1}, T_{2}\right) \sigma(s, u) d u d W(s) \\
& +\int_{0}^{t} \int_{\mathbb{R}} \int_{T_{1}}^{T_{2}} \widehat{w}\left(u ; T_{1}, T_{2}\right) \psi(s, u, z) d u \tilde{N}(d z, d s) .
\end{aligned}
$$

From uniqueness of the representation of a semimartingale, we find after comparing the coefficients,

$$
\begin{aligned}
F\left(0, T_{1}, T_{2}\right) & =\int_{T_{1}}^{T_{2}} \widehat{w}\left(u ; T_{1}, T_{2}\right) f(0, u) d u, \\
\Sigma\left(t, T_{1}, T_{2}\right) & =\int_{T_{1}}^{T_{2}} \widehat{w}\left(u ; T_{1}, T_{2}\right) \sigma(t, u) d u, \\
\Psi\left(t, T_{1}, T_{2}, z\right) & =\int_{T_{1}}^{T_{2}} \widehat{w}\left(u ; T_{1}, T_{2}\right) \psi(t, u, z) d u .
\end{aligned}
$$

This proves the Theorem. 
Proof of Theorem 4.4. This proof is based on the arguments of Prop. 13.1.1 in Musiela and Rutkowski, [44]. Since $S(t)=f(t, t)$, we have from (3.2)

$$
S(t)=S(0)+\int_{0}^{t} \sigma(s, t) d W(s)+\int_{0}^{t} \int_{\mathbb{R}} \psi(s, t, z) \tilde{N}(d z, d s) .
$$

The measurability and integrability conditions on $\sigma, \psi, \partial_{2} \sigma$ and $\partial_{2} \psi$ in Condition (F-S) allow us to use the stochastic Fubini Theorem in Protter [47, Thm. 46, p. 160] (or, after modifying the proof appropriately, Ikeda and Watanabe [31, Lem. 4.1, p. 116]),

$$
\begin{aligned}
\int_{0}^{t} \int_{\mathbb{R}} \psi(s, t, z) \tilde{N}(d z, d s) & =\int_{0}^{t} \int_{\mathbb{R}} \psi(s, s, z) \tilde{N}(d z, d s)+\int_{0}^{t} \int_{\mathbb{R}} \int_{s}^{t} \partial_{2} \psi(s, u, z) d u \tilde{N}(d z, d s) \\
& =\int_{0}^{t} \int_{\mathbb{R}} \psi(s, s, z) \tilde{N}(d z, d s)+\int_{0}^{t} \int_{0}^{u} \int_{\mathbb{R}} \partial_{2} \psi(s, u, z) \tilde{N}(d z, d s) d u .
\end{aligned}
$$

The calculation for $\int_{0}^{t} \sigma(s, t) d W(s)$ is analogous.

Proof of Theorem 7.1. Under the swap measure, the proof becomes a routine calculation: The option payoff can be written as

$$
\max \left(0, F\left(T, T_{1}, T_{2}\right)-K\right)=\left(F\left(T, T_{1}, T_{2}\right)-K\right) \cdot I_{K}
$$

where the indicator function $I_{K}$ is

$$
I_{K}=\left\{\begin{array}{l}
1 \text { if } F\left(T, T_{1}, T_{2}\right) \geq K \\
0 \text { if } F\left(T, T_{1}, T_{2}\right)<K
\end{array}\right.
$$

The value of the option at time $t$ is given as

$$
\begin{aligned}
C(t) & =\mathrm{e}^{-r(T-t)} \mathbb{E}\left[\left[F\left(T, T_{1}, T_{2}\right)-K\right] \cdot I_{K} \mid \mathcal{F}_{t}\right] \\
& =\mathrm{e}^{-r(T-t)} \mathbb{E}\left[F\left(T, T_{1}, T_{2}\right) \cdot I_{K} \mid \mathcal{F}_{t}\right]-\mathrm{e}^{-r(T-t)} \mathbb{E}\left[K \cdot I_{K} \mid \mathcal{F}_{t}\right] .
\end{aligned}
$$

Now, consider the first term: By the Bayes Formula and the definition of the swap measure, we find

$$
\begin{aligned}
\mathbb{E}\left[F\left(T, T_{1}, T_{2}\right) \cdot I_{K} \mid \mathcal{F}_{t}\right] & =F\left(t, T_{1}, T_{2}\right) \mathbb{E}\left[\frac{F\left(T, T_{1}, T_{2}\right)}{F\left(t, T_{1}, T_{2}\right)} \cdot I_{K} \mid \mathcal{F}_{t}\right] \\
& =F\left(t, T_{1}, T_{2}\right) \mathbb{E}\left[\frac{L(T)}{L(t)} \cdot I_{K} \mid \mathcal{F}_{t}\right] \\
& =F\left(t, T_{1}, T_{2}\right) \frac{\mathbb{E}\left[L(T) \cdot I_{K} \mid \mathcal{F}_{t}\right]}{\mathbb{E}\left[L(T) \mid \mathcal{F}_{t}\right]} \\
& =F\left(t, T_{1}, T_{2}\right) \mathbb{E}_{Q^{\mathrm{SW}}\left[I_{K} \mid \mathcal{F}_{t}\right]}
\end{aligned}
$$

Hence, (7.4) follows. 


\section{REFERENCES}

[1] Adams, K. J., and van Deventer, D. R. (1994). Fitting yield curves and forward rate curves with maximum smoothness, Journal of Fixed Income, 52-62.

[2] Barlow, M. T. (2002). A diffusion model for electricity prices. Mathematical Finance, 12(4), 287-298.

[3] Barndorff-Nielsen, O. E. and Shephard, N. (2005). Financial volatility: Stochastic volatility and Lévy based models. Cambridge University Press.

[4] Benth, F.E., Ekeland, L., Hauge, R., and Nielsen, B.F. (2003). On arbitrage-free pricing of forward contracts in energy market. Applied Mathematical Finance, 10(4), pp. 325-336.

[5] Benth, F.E., Kallsen, J., and Meyer-Brandis, T. (2005). A non-Gaussian Ornstein-Uhlenbeck process for electricity spot price modeling and derivatives pricing. Preprint, University of Oslo.

[6] Bessembinder, H., Coughenour, J.F., Seguin, P.J., and Smoller M.M. (1996). Is there a term structure of futures volatilities? Reevaluating the Samuelson hypothesis, Journal of Derivatives, pp. 45-58.

[7] Bessembinder, H. and Lemmon, M. (2002). Equilibrium pricing and optimal hedging in electricity forward markets. Journal of Finance, 57, 1347-1382.

[8] Black, F. (1976). The pricing of commodity contracts. Journal of Financial Economics, 3, pp. 167-179.

[9] Bjerksund, P., Rasmussen, H. and Stensland, G. (2000). Valuation and risk management in the Nordic electricity market. Working paper, Institute of finance and management sciences, Norwegian School of Economics and Business Administration.

[10] Bjørk, T. and Landén, C. (2002). On the term structure of futures and forward prices. Mathematical Finance - Bachelier Congress 2000, Geman, Madan, Pliska and Ton Vorst (eds.), pp. 111-150, SpringerVerlag.

[11] Burger, M., Klar, B., Müller, A., and Schindlmayr, G. (2004). A spot market model for pricing derivatives in electricity markets. Quantitative Finance, 4, pp. 109-122.

[12] Cartea, A. and Figueroa, M. G. (2004). Pricing in electricity markets: a mean reverting jump diffusion model with seasonality. Preprint, Birkbeck College, University of London.

[13] Clewlow, L. and Strickland, C. (2000). Energy Derivatives: Pricing and Risk Management. Lacima Publications.

[14] Da Prato, G. and Zabczyk, J. (1992). Stochastic Equations in Infinite Dimensions. Encyclopedia of Mathematics and its Applications, 44. Cambridge University Press, Cambridge.

[15] Deng, S. (2000). Stochastic models of energy commodity prices and their applications: mean reversion with jumps and spikes, Working paper, PWP-073 of the Program on Workable Energy Regulation (POWER).

[16] Escribano, A., Pena, J. I. and Villaplana, P. (2002). Modelling electricity prices: International evidence, Universidad Carlos III de Madrid working paper.

[17] Elliot, R. J., Sick, G. A. and Stein, M. (2000). Pricing electricity calls. Preprint, University of Alberta.

[18] Fabra, N., von der Fehr, N-H., Harbord, D. (2004). Designing Electricity Auctions, Center for the Study of Energy Markets (CSEM) Working Paper No. 122.

[19] Fackler, P.L., and Tian, Y. (1999). Volatility models for commodity markets, Working Paper, North Carolina Sate University.

[20] Fleten, S. and Lemming, J. (2003). Constructing forward price curves in electricity markets, Energy Economics, Forthcomming.

[21] Galloway, T.M., and Kolb, R.W. (1996). Futures prices and the maturity effect, Journal of Futures Markets, 16, pp. 809-828.

[22] Geman, H., El Karoui, N. and Rochet, J. C. (1995). Changes of numéraire, changes of probability measure and option pricing. Journal of Applied Probability, 32, pp. 385-408. 
[23] Geman, H., Roncoroni, A. (2006). Understanding the fine structure of electricity prices, The Journal of Business, forthcomming in $\mathbf{7 9}(5)$.

[24] Guthrie, G., and Videbeck, S. (2002). High frequency electricity spot price dynamics: An intraday markets approach, Working Paper, New Zealand Institute for the Study of Competition and Regulation.

[25] Harvey, A.C. (1990). Forecasting, Structural Time Series Models and the Kalman Filter. Cambridge University Press, Cambridge.

[26] Heath, D., Jarrow, R. and Morton, A. (1992). Bond pricing and the term structure of interest rates: a new methodology for contingent claim valuation. Econometrica, 60, pp. 77-105.

[27] Hinz, J. (2003). Modelling day-ahead prices. Applied Mathematical Finance, 10(2),149-161.

[28] Hinz, J., von Grafenstein, L., Verschuere, M., and Wilhelm, M. (2004). Pricing electricity risk by interest rate methods. Working paper.

[29] Hjalmarson, E. (2003). Does the Black-Scholes formula work for electricity markets? A nonparametric approach. Göteborg University, Working Papers in Economics, nr. 101.

[30] Hull, J. C. (2000). Options, Futures, and other derivatives. Prentice Hall.

[31] Ikeda, N. and Watanabe, S. (1981). Stochastic Differential Equations and Diffusion Processes. NorthHolland/Kodansha.

[32] Kaaresen, K. and Husby, E. (2002). A joint state-space model for electricity spot and futures prices. EPRM.

[33] Karatzas, I. and Shreve, S. E. (1991). Brownian Motion and Stochastic Calculus. Springer-Verlag.

[34] Kellerhals, P. (2001). Pricing electricity forwards under stochastic volatility, Working Paper, University of Tübingen.

[35] Keppo, J., Audet, N., Heiskanen, P. and Vehvilinen, I. (2004). Modeling electricity forward curve dynamics in the Nordic Market. In Modelling Prices in Competitive Electricity Markets. Bunn, D. W. (eds.), Wiley Series in Financial Economics, pp. 251-264.

[36] Knittel, C.R. and Roberts M. (2001). An empirical examination of deregulated electricity prices, POWER WP-087, University of California Energy Institute.

[37] Koekebakker, S. and Lien, G. (2004). Volatility and Price Jumps in Agricultural Futures Prices Evidence from Wheat Options, American Journal of Agricultural Economics, 86(4), pp. 1018-1031.

[38] Koekebakker, S. and Ollmar, F. (2005). Forward curve dynamics in the Nordic electricity market, Managerial Finance, 31(6), pp. 74-95.

[39] Kristiansen, T. (2004). Pricing of Contracts for Differences in the Nordic Market. Energy Policy, 32, pp. 1075-1085.

[40] Leon, A. and Rubia, A. (2004). Testing for weekly seasonal unit roots in the Spanish power pool, In Modelling Prices in Competitive Electricity Markets. Bunn, D. W. (eds.), Wiley Series in Financial Economics, pp. 177-189.

[41] Longstaff, F. A. and Wang, A.W. (2004). Electricity Forward Prices: A High-Frequency Empirical Analysis. Journal of Finance, 59(4), pp. 1877 - 1900.

[42] Lucia, J. and Schwartz, E. S. (2002). Electricity Prices and Power Derivatives: Evidence from the Nordic Power Exchange. Review of Derivatives Research, 5(1), pp. 5-50.

[43] Merton, R. (1976). Option pricing when underlying stock returns are discontinuous. Journal of Financial Economics, 3, pp. 125-144.

[44] Musiela, M. and Rutkowski, M. (1998). Martingale Methods in Financial Modeling. Springer-Verlag.

[45] Ollmar, F. (2003). Smooth forward price curves in electricity markets. Ch. 1 in An analysis of derivatives prices. Ph.D. dissertation at the Norwegian School of Economics and Business Administration, Norway. 
[46] Pilipovic, D. (1998). Energy Risk: Valuing and managing energy derivatives. Mc Graw-Hill, New York.

[47] Protter, Ph. (1990). Stochastic Integration and Differential Equations. Springer-Verlag.

[48] Saravia, C. (2003). Speculative Trading and Market Performance: The Effect of Arbitrageurs on Efficiency and Market Power in the New York Electricity Market, Center for the Study of Energy Markets (CSEM), Working Paper No 121.

[49] Samuelson, P. (1965). Rational theory of warrant pricing. Indust. Management Rev., 6, pp. 13-32.

[50] Schwartz, E. S. (1997). The stochastic behaviour of commodity prices: Implications for valuation and hedging. J. Finance, LII(3), pp. 923-973.

[51] Shiryaev, A. N. (1999). Essentials of Stochastic Finance. World Scientific.

[52] Skantze, P., Gubina, A., and Ilic, M. (2000). Bid-based stochastic model for electricity prices: the impact of fundamental drivers on market dynamics Report MIT Energy Laboratory

[53] Skantze, P. and Ilic, M. (2000). The joint dynamics of electricity spot and forward markets: implications on formulating dynamic hedging strategies Report MIT Energy Laboratory.

[54] Stoft, S., (2002). Power System Economics, John Wiley and Sons, New York, NY.

[55] Strickland L. (2002). Derivative modelling approaches used in real option valuation, Manuscript presented at Optimal Methods for Applying Real Options in the Energy Industry, London, UK, 4-5 November.

[56] Vehviläinen, I. and Pyykkönen, T. (2004). Stochastic factor model for the electricity spot price - the case of the Nordic market. Preprint, Helsinki University of Technology.

[57] White, H. (1982). Maximum likelihood estimation of misspecified models, Econometrica, 50(1). pp. $1-25$.

[58] Wolak, F. (1997). Market design and price behavior in restructured electricity markets: an international comparison, POWER WP-051, University of California Energy Institute.

(Fred Espen Benth)

Centre of Mathematics for Applications

Department of Mathematics

UNIVERSITY OF OSLO

P.O. Box 1053, BLINDERN

N-0316 Oslo, Norway

AND

Agder University College

School of Management

Serviceboks 422

N-4604 Kristiansand, Norway

E-mail address: fredb@math .uio.no

$U R L:$ http://www.math.uio.no/ fredb/

(Steen Koekebakker)

Agder University College

SCHOOL OF MANAGEMENT

Serviceboks 422

N-4604 Kristiansand, Norway

E-mail address: steen.koekebakker@hia .no 